\title{
Precipitation Estimation from Remotely Sensed Imagery Using an Artificial Neural Network Cloud Classification System
}

\author{
YANG HONG \\ Department of Hydrology and Water Resources, The University of Arizona, Tucson, Arizona, and Department of Civil and Environmental \\ Engineering, University of California, Irvine, Irvine, California \\ KuO-Lin Hsu \\ Department of Civil and Environmental Engineering, University of California, Irvine, Irvine, California \\ SOROOSH SOROOSHIAN \\ Department of Hydrology and Water Resources, The University of Arizona, Tucson, Arizona, and Department of Civil and Environmental \\ Engineering, University of California, Irvine, Irvine, California \\ XIAOGANG GaO \\ Department of Civil and Environmental Engineering, University of California, Irvine, Irvine, California
}

(Manuscript received 16 September 2003, in final form 17 June 2004)

\begin{abstract}
A satellite-based rainfall estimation algorithm, Precipitation Estimation from Remotely Sensed Information using Artificial Neural Networks (PERSIANN) Cloud Classification System (CCS), is described. This algorithm extracts local and regional cloud features from infrared $(10.7 \mu \mathrm{m})$ geostationary satellite imagery in estimating finescale $\left(0.04^{\circ} \times 0.04^{\circ}\right.$ every $\left.30 \mathrm{~min}\right)$ rainfall distribution. This algorithm processes satellite cloud images into pixel rain rates by 1 ) separating cloud images into distinctive cloud patches; 2) extracting cloud features, including coldness, geometry, and texture; 3) clustering cloud patches into well-organized subgroups; and 4) calibrating cloud-top temperature and rainfall $\left(T_{b}-R\right)$ relationships for the classified cloud groups using gauge-corrected radar hourly rainfall data. Several cloud-patch categories with unique cloud-patch features and $T_{b}-R$ curves were identified and explained. Radar and gauge rainfall measurements were both used to evaluate the PERSIANN CCS rainfall estimates at a range of temporal (hourly and daily) and spatial $\left(0.04^{\circ}, 0.12^{\circ}\right.$, and $\left.0.25^{\circ}\right)$ scales. Hourly evaluation shows that the correlation coefficient (CC) is $0.45(0.59)$ at a $0.04^{\circ}\left(0.25^{\circ}\right)$ grid scale. The averaged CC of daily rainfall is $0.57(0.63)$ for the winter (summer) season.
\end{abstract}

\section{Introduction}

Reliable observation of precipitation is an important task to the hydrologic and climate research communities. Because ground observation from gauge and radar suffers from spatial and temporal gaps, rainfall measurements derived from meteorological satellites become an attractive option because of their high spatial and temporal sampling frequencies.

In our earlier reported work, we developed the Precipitation Estimation from Remotely Sensed Information using Artificial Neural Networks (PERSIANN) algorithm, using satellite longwave infrared (IR) images (Hsu et al. 1997, 1999; Sorooshian et al. 2000). The PERSIANN algorithm fits the pixel brightness temper-

Corresponding author address: Yang Hong, Center for Hydrometeorology and Remote Sensing, Department of Civil and Environmental Engineering, E-4130 Engineering Gateway, University of California, Irvine, Irvine, CA 92697-2175.

E-mail: yanghong@uci.edu ature and its neighbor temperature textures, in terms of means and standard deviations, to the calculated pixel rain rates based on an Artificial Neural Network (ANN) model. The PERSIANN algorithm is operated at a spatial resolution of $0.25^{\circ}$ latitude $\times 0.25^{\circ}$ longitude, with model parameters routinely adjusted by other low-orbital satellite microwave rainfall estimates (Sorooshian et al. 2000).

Improvement of the PERSIANN system for estimating rainfall rates at higher spatial and temporal resolution is ongoing. In this paper, the development of the PERSIANN Cloud Classification System (CCS) is introduced. Instead of direct pixel-to-pixel fitting of infrared cloud images to the rain rate, the PERSIANN CCS takes into account image segmentation and objective classification methods to process cloud images into a set of disjointed cloud-patch regions; informative features are extracted from cloud patches and classified into a number of patch groups based on the similarity of selected features, such as the patch size and texture. 


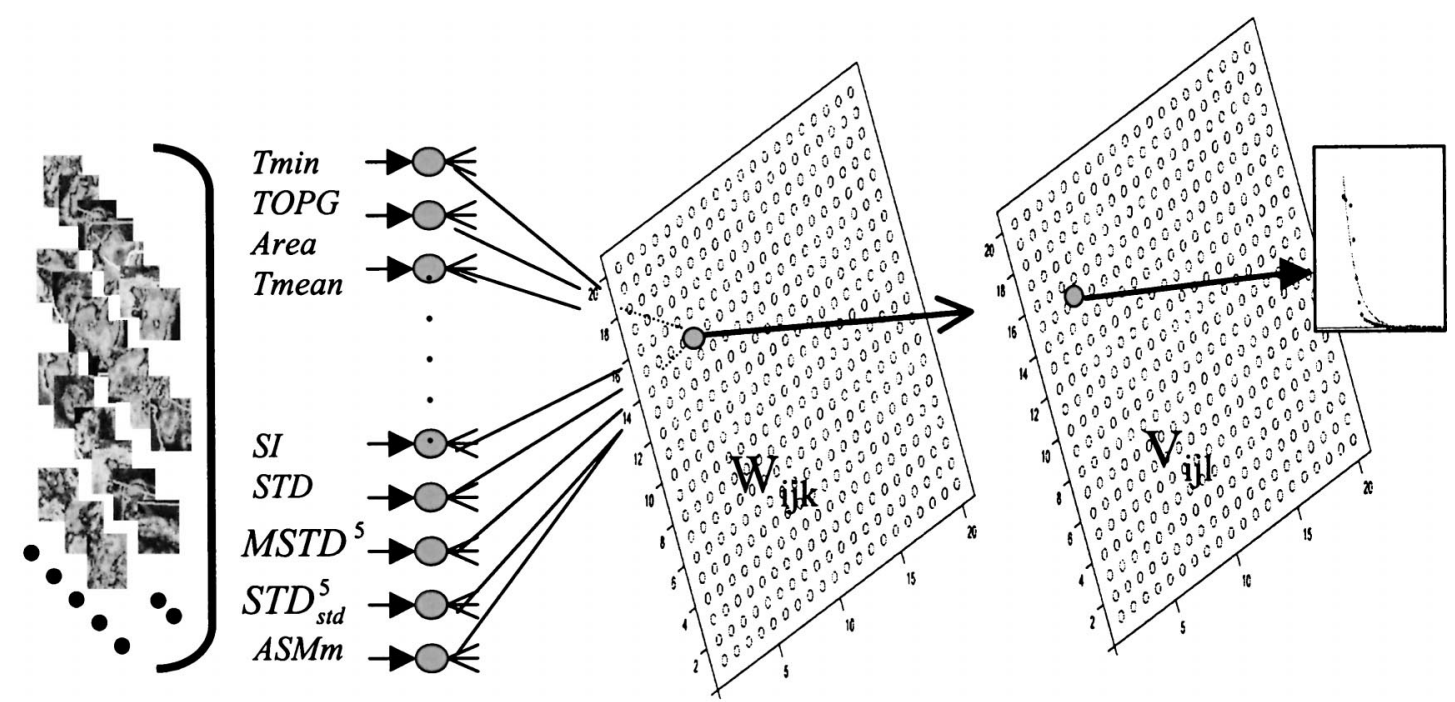

\section{$\begin{array}{lll}\text { (a) Cloud Segmentation (b) Feature Extraction } & \text { (c) Cloud Classification } & \text { (d) Rainfall Mapping }\end{array}$}

FIG. 1. The PERSIANN CCS model structure: (a) image preprocessing through a cloud segmentation procedure, (b) input feature extraction of the cloud patch, (c) cloud-patch feature classification $\left(20 \times 20\right.$ clusters), and (d) nonlinear temperature and rainfall $\left(T_{b}-R\right)$ fitting for the $20 \times 20$ classified cloud clusters, with one fitting curve per cluster. Note that $W$ and $V$ are the connection weights for the SOFM layer and multiparameter nonlinear fitting layer, respectively. The final output of this network is the calibrated $T_{b}-R$ for every classified cloud cluster.

Pixel rain rates under the cloud-patch coverage are assigned using the histogram-matching method and exponential regression fitting of pixel brightness temperatures $T_{b}$ and rainfall rates $R$. Each classified cloud-patch group is specified a unique temperature-rainfall $\left(T_{b}-R\right)$ curve, and the parameters of the $T_{b}-R$ curve are calibrated based on rain estimates of the Next-Generation Weather Radar (NEXRAD) network.

The scope of this paper is as follows. Section 2 describes algorithms in rainfall estimation using infrared imagery. The structure of PERSIANN CCS is presented in section 3, followed by the network training and some insights provided by PERSIANN CCS calibration in section 4 . Section 5 presents validation results, and section 6 discusses conclusions and suggestions for further improvements to PERSIANN CCS.

\section{Background}

Satellite IR-based rainfall estimation algorithms offer unique advantages of extensive global coverage at relatively high temporal sampling rates (Ebert and Manton 1998). The algorithms developed to date may be classified into three groups, depending on the level of information extracted from the infrared cloud images. There are

1) cloud-pixel-based algorithms,

2) cloud-local-texture-based algorithms, and

3) cloud-patch-based algorithms.

An example of the cloud-pixel-based algorithm is the
Geostationary Operational Environmental Satellite (GOES) precipitation index (GPI; Arkin and Meisner 1987), which assigns a constant conditional rain rate of $3 \mathrm{~mm} \mathrm{~h}^{-1}$ to pixels with a $T_{b}$ lower than $235 \mathrm{~K}$ and zero rain rates otherwise. The adjusted GPI (AGPI; Adler and Negri 1993) adjusts rain rate by time- and spacematched GPI and Special Sensor Microwave Imager (SSM/I)-based rain estimates accumulated for a month. $\mathrm{Xu}$ et al. (1999a) proposed a universal AGPI that is similar to AGPI but allows both the rain rate and temperature threshold to vary. Another pixel-based fitting is the autoestimator (AE) algorithm (Vicente et al. 1998). The AE algorithm utilizes a power-law function to fit the $T_{b}-R$ relationship. Pixel rainfall values are further adjusted using several correction factors, such as relative humidity and precipitable water.

Cloud-local-texture-based approaches retrieve pixel rain rates by extending the mapping from one single pixel to a range of the neighborhood pixel coverage. Wu et al. (1985) used 24 brightness temperature texture features to retrieve rainfall within a neighborhood size of $20 \mathrm{~km} \times 20 \mathrm{~km}$. Hsu et al. (1997) developed the PERSIANN system, which calculates rain rate at $0.25^{\circ}$ latitude $\times 0.25^{\circ}$ longitude resolution, based on the brightness temperature variations in the neighboring coverage of $1.25^{\circ} \times 1.25^{\circ}$.

Cloud-patch-based approaches estimate rainfall based on the cloud coverage under a specified temperature threshold, such as $253 \mathrm{~K}$. The Griffith-Woodley Technique (Griffith et al. 1978; Woodley et al. 1980) tracked 


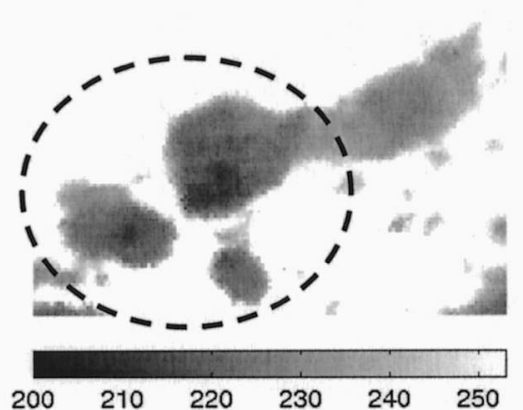

(a) Infrared cloud image

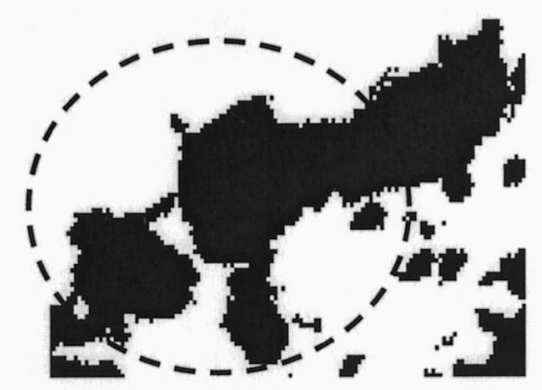

(b) Fixed threshold (253K) segmentation of cloud image

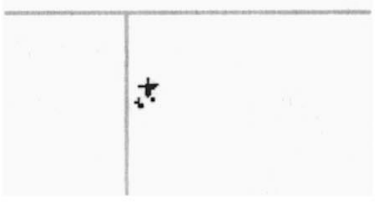

(c) $212 \mathrm{~K}$

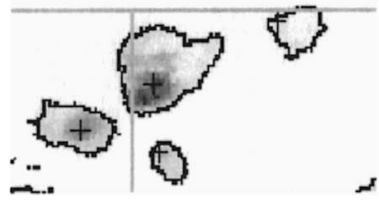

(f) $230 \mathrm{~K}$

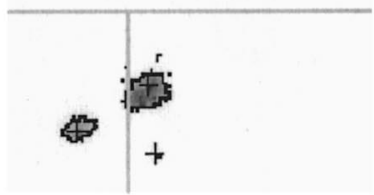

(d) $218 \mathrm{~K}$

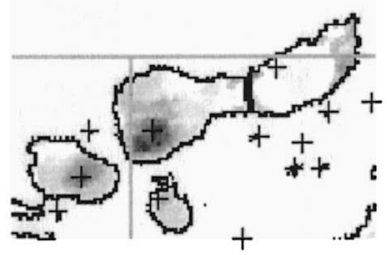

(g) $242 \mathrm{~K}$

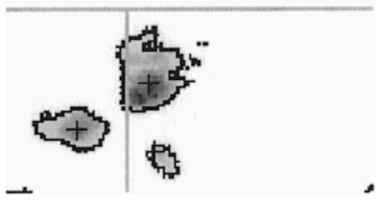

(e) $2224 \mathrm{~K}$

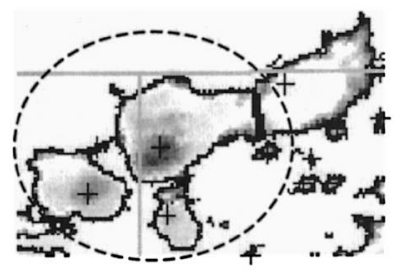

(h) $253 \mathrm{~K}$

FIG. 2. (a) The IR cloud image, (b) segmentation of the cloud image, using the fixed threshold of $253 \mathrm{~K}$, and (c)-(h) the cloud-patch segmentation process using the ITT algorithm in this study.

\section{TABLE 1. Input features extracted from cloud patches.}

Coldness features of cloud patch

1) Min temperature of a cloud patch $\left(T_{\min }\right)$

2) Mean temperature of a cloud patch $\left(T_{\text {mean }}\right)$

Geometric features

3) Cloud-patch area (AREA)

4) Cloud-patch shape index (SI)

Texture features (referring to the brightness temperature texture)

5) Std dev of cloud-patch temperature (STD)

6) Mean value of local std dev of cloud temperature $\left(\mathrm{MSTD}^{5}\right)$

7) Std dev of local std dev of cloud $T_{b}\left(\mathrm{STD}_{\mathrm{std}}^{5}\right)$

8) Gradient of cloud-top brightness temperature (TOPG)

9) Gray-image texture (maximum angular second moment) a cloud through its lifetime. The total rain volume of the cloud patch is determined based on the ratios of satellite cloud area to its maximum coverage. The Convective-Stratiform Technique (Adler and Negri 1988) is another example of the cloud-patch-based approach. It screens convective cells based on the local minimum of infrared temperature and assigns different rainfall amounts to convective and stratiform components separately. Pixel rain rates are proportionally distributed starting from the coldest pixel to higher-temperature pixels. Xu et al. (1999b) proposed another approach, which determines different temperature thresholds by separating the rain/no-rain pixels in a cloud patch using $\mathrm{SSM} / \mathrm{I}$ microwave rainfall estimates.

With respect to the use of information, both pixeland local-texture-based approaches only utilize limited attributes of the cloud patches. Rain rates retrieved from 

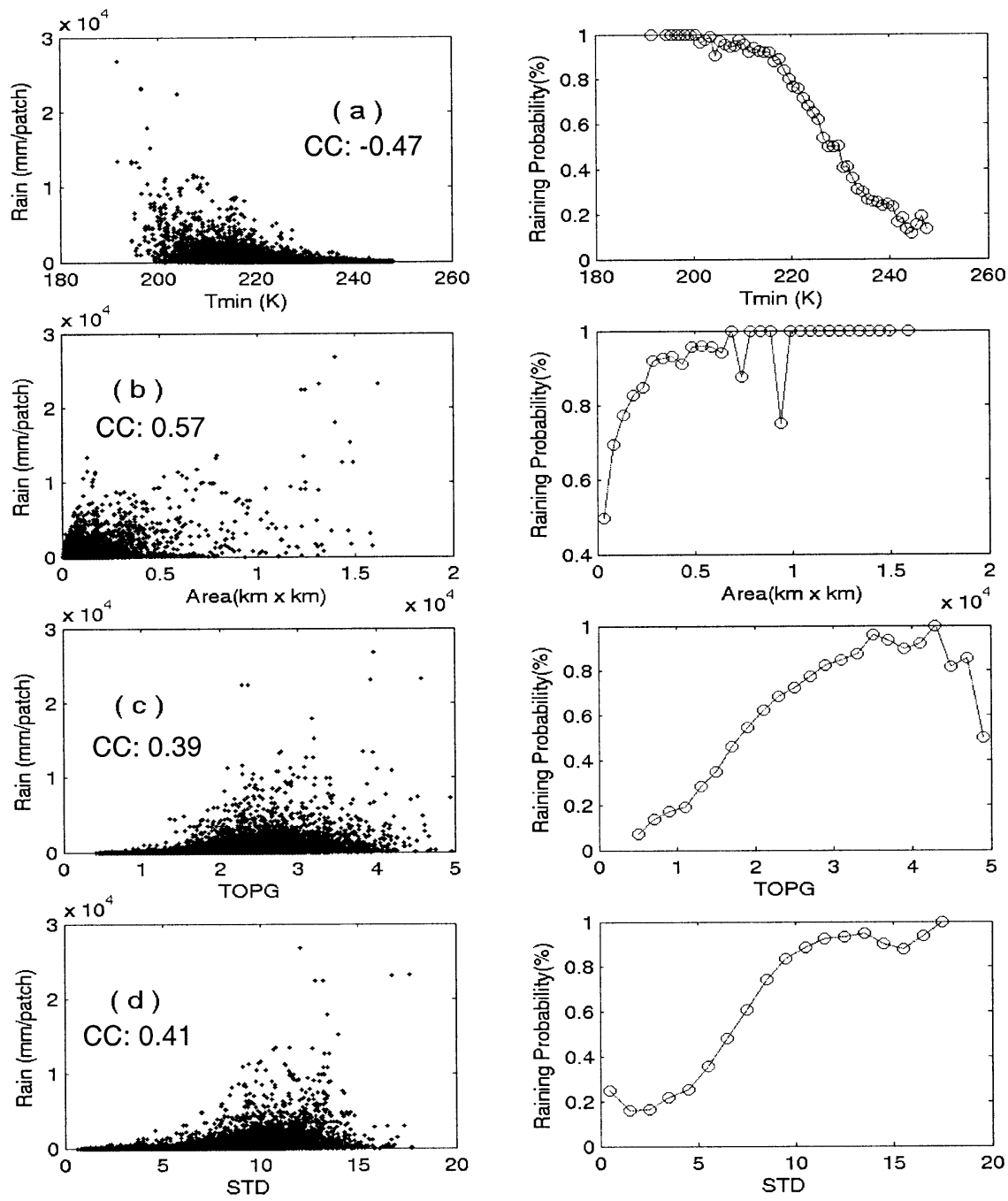

FIG. 3. Cloud-patch features of $T_{\min }$, AREA, TOPG, and STD vs patch rainfall and patch rain probability. Note that not all of the selected features are shown here. (left) Values of cloud-patch features vs cloud-patch rainfall, and (right) interval-averaged values of cloud-patch features vs patch rain probability.

these methods tend to be nonunique and may be insufficient to identify the relationships between cloud types and surface rain rates. Cloud-patch-based approaches, on the other hand, attempt to include more information from the cloud images and are likely to provide a more reliable rainfall retrieval system than do the pixel-based approaches. Therefore, successful characterization of cloud patches can be one step toward better estimation of rainfall. However, developing a desirable cloudpatch-based algorithm depends on many factors, including 1) how effectively the cloud image may be separated into distinct cloud systems or cells; 2) how our knowledge of the cloud systems may be converted into a set of measurable numerical feature vectors, in terms of patch temperature, size, and texture; 3) how these feature vectors may be effectively clustered into a set of separable cloud-patch "classes" or "groups;" and 4) how the cloud-patch groups are associated with the rainfall distributions under the cloud coverage. Following the above requirements, the PERSIANN CCS is developed. A description of the algorithm is listed in the following section.

\section{Method}

The PERSIANN CCS establishes different $T_{b}-R$ relationships, calibrated by collocated cloud images and gauge-corrected radar rainfall data, for every classified cloud group by implementing a sequence of four steps (Fig. 1). The steps are described as follows.

\section{a. Segmentation of satellite infrared cloud images}

A cloud image is shown in Fig. 2a, and cloud-patch segmentation of this image, using a fixed threshold (253 


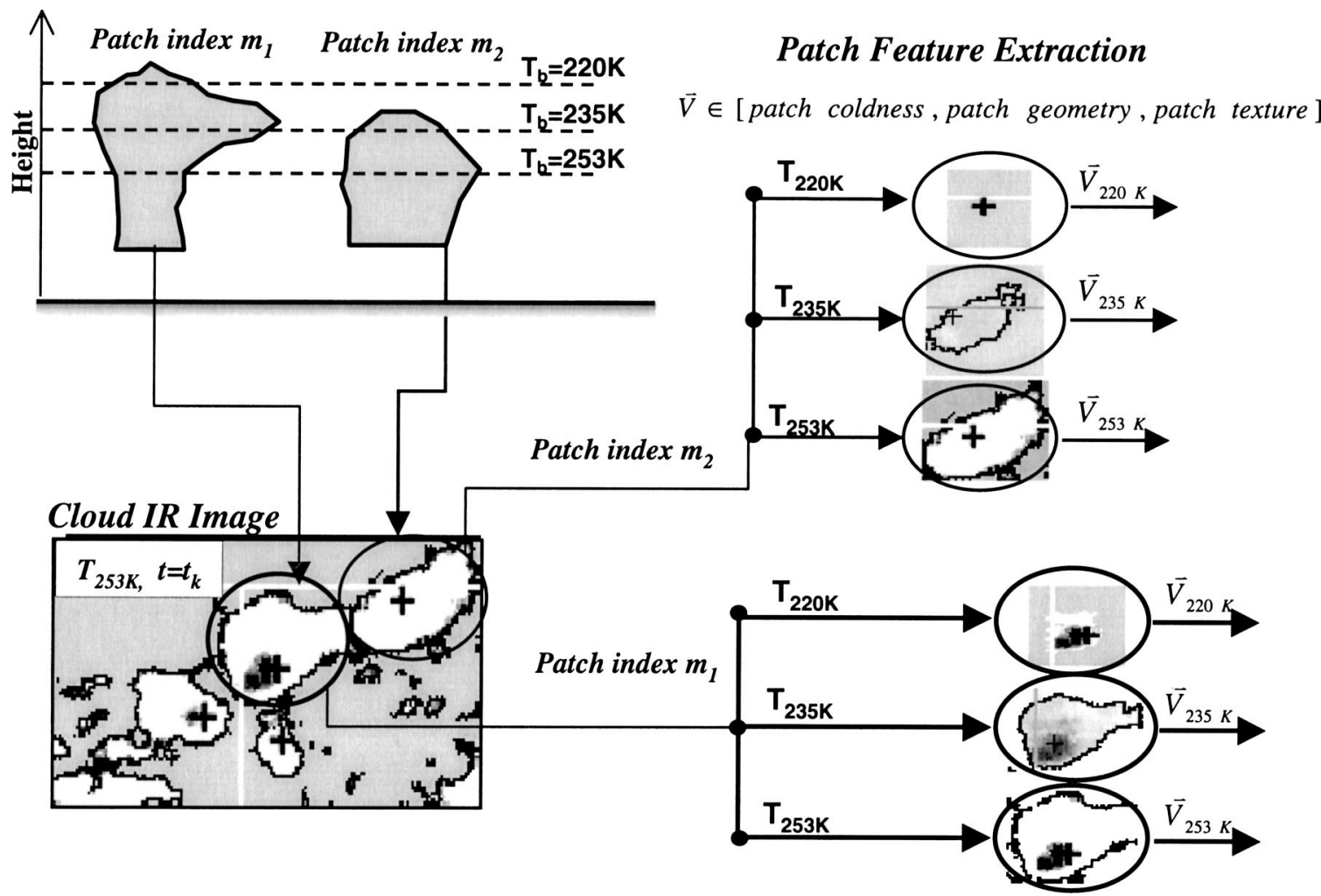

FIG. 4. Cloud-patch features calculated at three separate temperature levels (220, 235, and $253 \mathrm{~K})$.

$\mathrm{K})$, is shown in Fig. 2b. Note that even though the cloud image contains several convective cells, the single threshold cannot separate them effectively.

Our proposed approach, called the incremental temperature threshold (ITT), achieves segmentation by gradually increasing threshold temperatures (Figs. 2ch). The algorithm (for a complete description of ITT, please see appendix A) locates the local minimum temperature $T_{\min }$ and places the first set of seeds (illustrated as cross markers in Fig. 2). Next, the threshold temperature is raised and extended to the neighboring areas from the seeded points until the border of other seeded regions or cloud-free regions is reached.

\section{b. Extraction of cloud-patch features}

Fully developed convective clouds have distinguishable overshooting tops, tight temperature gradients, and higher local pixel temperature variations. Stratiform clouds, on the other hand, show more gradual temperature gradients and lower temperature variations. The three categories used to discriminate between cloud types are coldness, geometry, and texture. A further breakdown of measurable features is provided in Table 1 , and a detailed explanation of these features is listed in appendix B.

As one might expect, there is a potential interrelationship between the selected cloud-patch features and the amounts of patch rainfall. Figure 3 illustrates the scatterplots of the four selected features with respect to hourly patch rainfall (millimeters per patch) and patch rain probability, based on 1-month (June 1999) GOES images and gauge-corrected radar-rainfall data over the region of $30^{\circ}-40^{\circ} \mathrm{N}$ and $120^{\circ}-100^{\circ} \mathrm{W}$. Note that the rain probability is the ratio of the rain patches $(\geq 10$ millimeters per patch) to the total number of patches within a small interval of displayed feature. The left-hand panels show a negative correlation trend with respect to the minimum temperature (Fig. 3a) and positive correlation for the remaining three features (Figs. 3b-d). The widescattering samples in the figures are partly due to the interaction among the patch features. In the case of Fig. $3 \mathrm{a}$, for example, the amount of patch rainfall is not only determined by $T_{\min }$, but is also influenced by other factors, such as the patch size and texture. In the case of the rain probability plots depicted in the right-hand panels of Fig. 3, we witness a monotonically decreasing trend in the case of $T_{\min }$ (i.e., a lower patch minimum temperature is related to a higher probability of rain in the patch) and increasing trends for the other three features.

\section{c. Classification of cloud patches}

A clustering algorithm, the self-organizing feature map (SOFM), is used to classify the cloud-patch features into a number of groups (Kohonen 1982). This approach 
(a) $20 \times 20$ SOFM classified groups of cloud patches

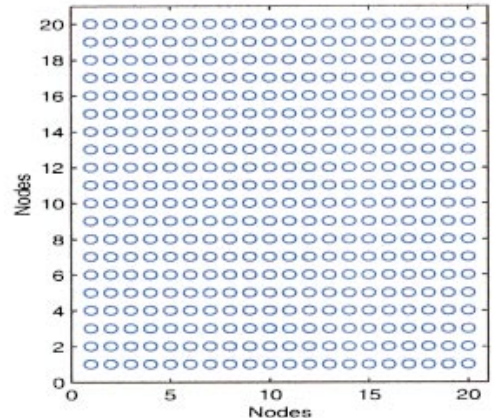

(b) $T_{\min }(K)$

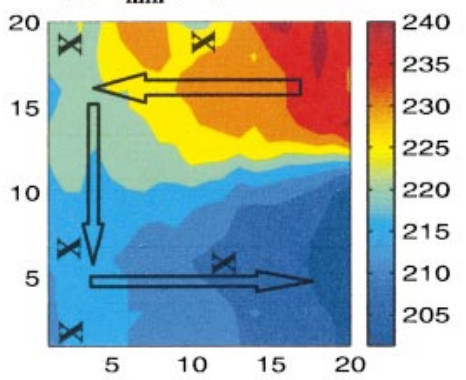

(e) Rain rate $(\mathrm{mm} / \mathrm{hr})$

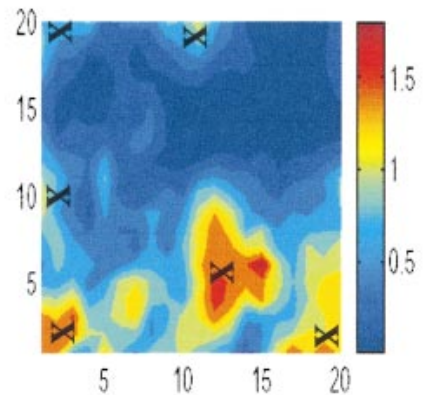

(c) TOPG

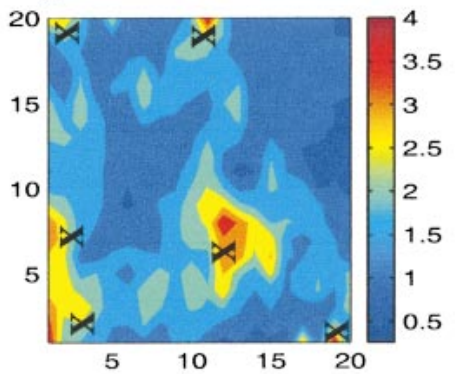

(f) Area (pixel)

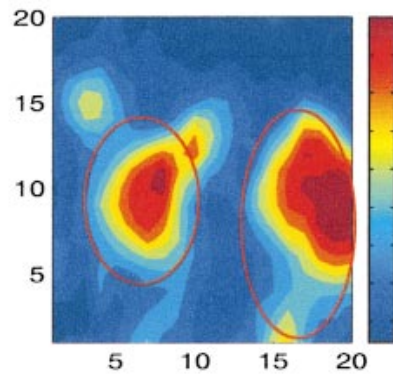

(d) MSTD M $^{5}$

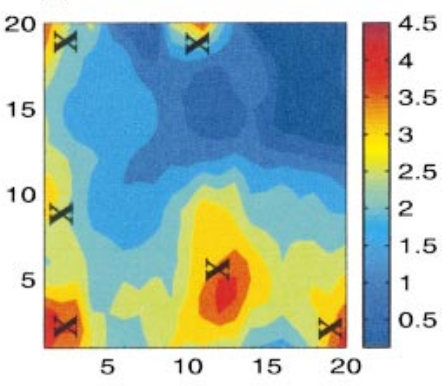

(g) Patch rainfall ( $\mathrm{mm} / \mathrm{patch})$

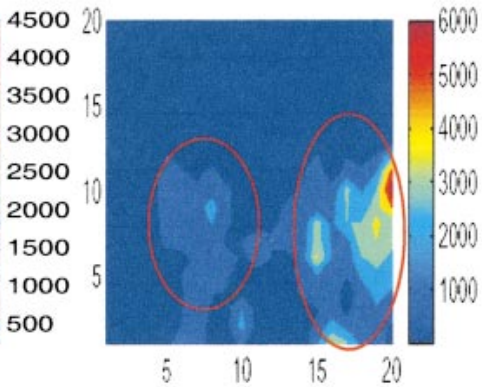

FIG. 5. (a) The SOFM classified $20 \times 20$ nodes (groups) of cloud patches and (b) $-(\mathrm{g})$ their associated feature maps and rainfall by training using the Jun 1999 GOES IR and radar rainfall data.

has already been described in our earlier work (Hsu et al. 1997), and therefore only a brief description is provided here. SOFM projects patterns of high-dimensional space to lower-dimensional space. The projection enables the classification of input patterns of many variables into a number of clusters arranged in a two-dimensional coordinate (Fig. 1c). The two main steps involved are

1) calculating the distance between patch features and the SOFM cluster center using

$$
d_{j}=\left[\sum_{i=1}^{n_{1}}\left(x_{i}-w_{i j}\right)^{2}\right]^{1 / 2}, \quad j=1, \ldots, n,
$$

where $x_{i}$ is the cloud-patch input feature $i$, and $w_{i j}$ is the connection parameter of SOFM from input feature $i$ to the specified cluster center (node) $j$, and 2) finding the best matching SOFM cluster center $c$, from the minimum distance between the input feature vector and the SOFM connection weights $w_{i j}$ :

$$
d_{c}=\min \left(d_{j}\right), \quad \text { where } j=1, \ldots, n .
$$

\section{d. Estimation of patch and pixel rainfall}

The relationship of pixel temperature $T_{b}$ and surface rainfall $R$ of a cloud patch is likely to vary in different stages of its life cycle. Therefore, we assign different $T_{b}-R$ relationships to various cloud patches based on the classification resulting from the previous clustering stage. 
(a) The calibrated $20 \times 20 \mathrm{~T}_{\mathrm{b}}-\mathrm{R}$ curves after SOFM classification

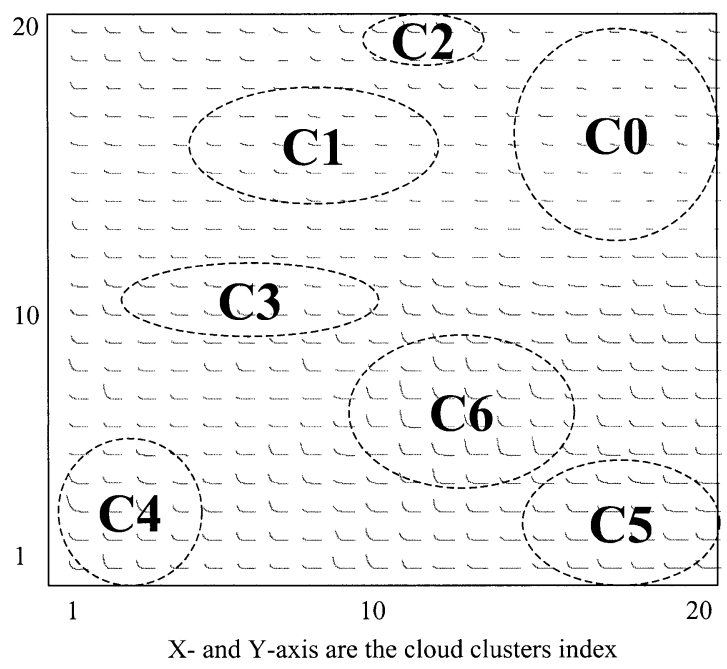

(b) Typical $T_{b}-R$ curves from the 6 clusters in Figure $5 \mathrm{a}$

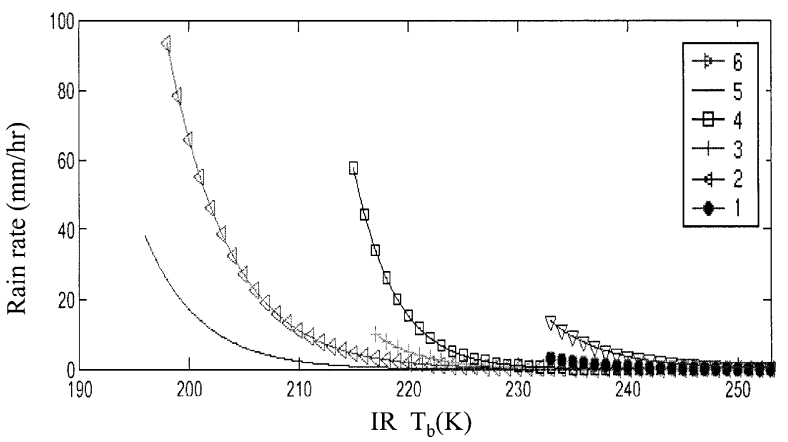

FIG. 6. (a) The calibrated cloud-rainfall relationships (400 $T_{b}-R$ curves) from the PERSIANN CCS algorithm using IR and radar data, with each curve referring to a cloud cluster in the SOFM classification map (Fig. 5a.). (b) Several typical $T_{b}-R$ curves from clusters in (a). Note that the curve of cluster 0 does not show in (b) because it is short and flat.

In each classified cloud-patch group, the $T_{b}-R$ pixel pairs are first redistributed using the probability matching method (Atlas et al. 1990). This method matches histograms of $T_{b}$ and $R$ observations such that the proportion of the $R$ distribution above a given rain rate is equal to the proportion of the $T_{b}$ distribution below the associated $T_{b}$ threshold value. The redistributed pixels are fitted with a nonlinear exponential function for each patch group. Given SOFM-classified patch group $j$, the $T_{b}-R$ relationship is specified as

$$
R^{j}=v_{1}^{j}+v_{2}^{j} \exp \left[v_{3}^{j}\left(T_{b}+v_{4}^{j}\right)^{v_{5}^{j}}\right],
$$

where $R$ is the rainfall rate $\left(\mathrm{mm} \mathrm{h}^{-1}\right), T_{b}$ is the cloudtop brightness temperature (K), and $v_{1}^{j}, v_{2}^{j}, v_{3}^{j}, v_{4}^{j}$, and $v_{5}^{j}$ are parameters with respect to patch group $j$. The parameters of the $T_{b}-R$ functions in each patch group are calibrated using a large number of GOES infrared images and their collocated gauge-corrected radar rainfall.

\section{PERSIANN CCS calibration}

\section{a. System architecture and calibration}

To implement the PERSIANN CCS, we first need to specify the number of cloud-patch groups. There is a trade-off between the number of cloud-patch groups and the accuracy and efficiency of the calibrated model. To ensure good accuracy, a large number of classified cloud groups are required, but the computational requirements for model training and testing increase dramatically. Hsu et al. (2002) show that, given an objective function (root-mean-square error, e.g.), an optimal group size can be found by progressively testing the size of groups. In this study, a group size $(20 \times 20)$ was determined in the SOFM classification layer.

The nine input features listed in Table 1, except $T_{\min }$ and the gradient of the cloud-top brightness temperature TOPG, are calculated at three separate temperature levels $(220,235$, and $253 \mathrm{~K})$ for every cloud patch. Figure 4 is a demonstration of cloud feature extraction for two adjacent patches (shown as $m_{1}$ and $m_{2}$ ). Cloud $m_{1}$ depicts the situation of a patch in its mature stage with extensive vertical growth and an overshooting top, and patch $m_{2}$ is still in the towering stage with its top temperature not yet at the $220-\mathrm{K}$ level. Features extracted at the $220-\mathrm{K}$ level are related to the cold core of the taller cloud patch, while features extracted at the warmer $235-\mathrm{K}$ level represent information of larger extent. Patch features extracted from the threshold 253$\mathrm{K}$ level give information of the entire cloud patch ranging from $T_{\min }$ to $253 \mathrm{~K}$. However, not every cloud patch contains temperatures ranging up to the colder levels,

TABLE 2. Input features and precipitation characteristics of selected clusters on SOFM.

\begin{tabular}{|c|c|c|c|c|c|c|c|}
\hline Category & $T_{\min }(\mathrm{K})$ & Area (pixel) & TOPG & MSTD $^{5}$ & STD & $\begin{array}{l}\text { Pixel rain rate } \\
\text { (millimeters } \\
\text { per pixel) }\end{array}$ & $\begin{array}{l}\text { Patch rainfall } \\
\text { (millimeters } \\
\text { per patch) }\end{array}$ \\
\hline $\mathrm{C} 0$ & $>245$ & $<100$ & $<0.5$ & $<0.5$ & $<2$ & $<0.1$ & $<10$ \\
\hline $\mathrm{C} 1$ & $235-245$ & $100-1000$ & $\sim 1$ & 1 & $<4$ & $0-0.5$ & $<100$ \\
\hline $\mathrm{C} 2$ & $235-245$ & $100-1000$ & $\sim 3$ & 3.5 & $4-8$ & $0.5-0.1$ & $<1000$ \\
\hline $\mathrm{C} 3$ & $215-235$ & $500-4000$ & $\sim 1$ & 2.5 & $6-8$ & $0.1-0.5$ & $500-2000$ \\
\hline $\mathrm{C} 4$ & 215-235 & $500-4000$ & $\sim 3.5$ & 4 & $8-10$ & $1-1.5$ & $1000-2500$ \\
\hline $\mathrm{C} 5$ & $<215$ & $1500-5000$ & $\sim 1.5$ & 3 & $>10$ & $0.5-1.5$ & $3000-6000$ \\
\hline C6 & $<215$ & $1000-3500$ & $\sim 4.5$ & 5 & 8-14 & $1.5-4.5$ & $2000-3500$ \\
\hline
\end{tabular}


TABLE 3. Statistics for PERSIANN CCS and PERSIANN estimates of hourly rain rates under a range of spatial resolutions when compared with radar observation-a case study. Note: zero-rain-rate grids were included to compute the statistical information. Sample size for comparison is 1890 at $0.25^{\circ}$ grid resolution over the entire storm.

\begin{tabular}{|c|c|c|c|c|c|c|c|c|}
\hline \multirow[b]{2}{*}{ Resolution } & \multicolumn{4}{|c|}{ PERSIANN CCS } & \multicolumn{4}{|c|}{ PERSIANN } \\
\hline & Rmse & CORR & Ratio & CSI & Rmse & CORR & Ratio & CSI \\
\hline $0.04^{\circ} \times 0.04^{\circ}$ & 2.72 & 0.45 & 1.12 & 0.53 & - & - & - & - \\
\hline $0.12^{\circ} \times 0.12^{\circ}$ & 2.01 & 0.54 & 1.09 & 0.64 & - & - & - & - \\
\hline $0.25^{\circ} \times 0.25^{\circ}$ & 1.27 & 0.59 & 1.07 & 0.67 & 2.67 & 0.47 & 1.17 & 0.55 \\
\hline
\end{tabular}

such as 235 or $220 \mathrm{~K}$, where default values are assigned. For example, in the case of cloud-patch area, a zero is assigned for $T_{\min }$ at the $220-\mathrm{K}$ level for patch $m_{2}$, and it has a nonzero value for temperature levels of 235 and $253 \mathrm{~K}$.

After completing the cloud-patch feature extraction, the system is calibrated using GOES infrared images and radar-rainfall maps for June 1999 over the region of $25^{\circ}-45^{\circ} \mathrm{N}$ and $100^{\circ}-130^{\circ} \mathrm{W}$ (both datasets are mapped to $0.04^{\circ}$ latitude $\times 0.04^{\circ}$ longitude scale). The hourly rainfall used for calibration is a rain gauge-corrected multisensor precipitation dataset, developed by the National Oceanic and Atmospheric Administration National Centers for Environmental Prediction (NCEP) (NCEP 2003).

Calibration of the algorithm is a two-stage process. The first stage is an unsupervised clustering of input
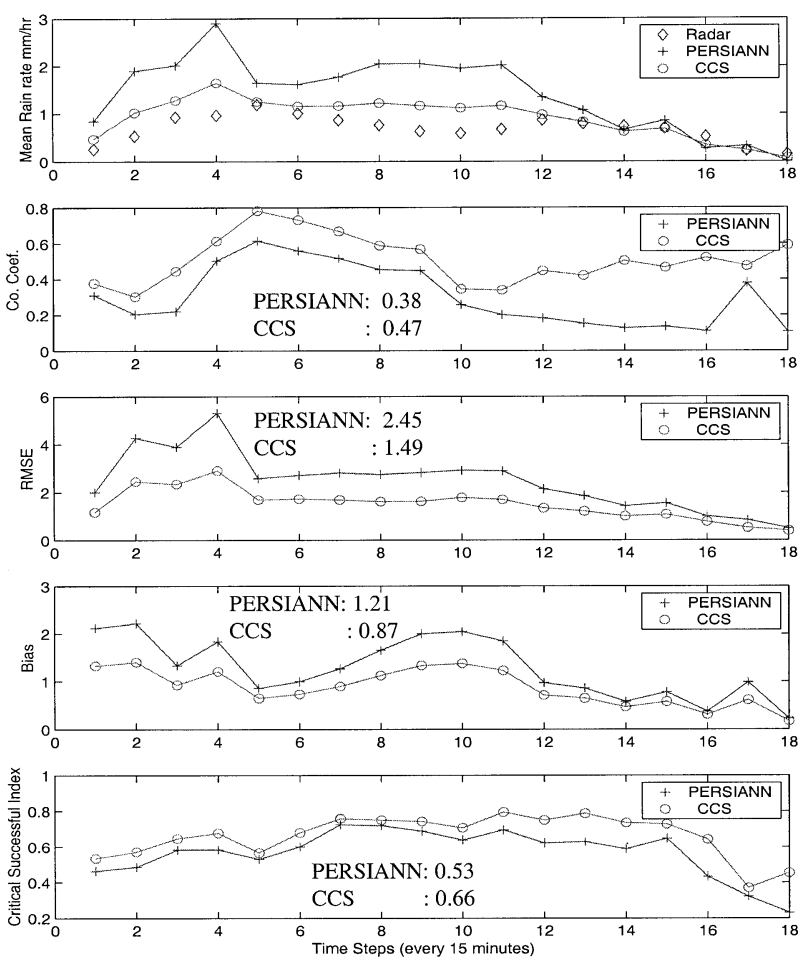

FIG. 7. Time series of statistics of hourly rain rates derived from PERSIANN CCS and PERSIANN over the Las Vegas, NV, vicinity flash-flooding storm from 1400 through 1900 UTC 8 Jul 1999. (The results were calculated at $0.25^{\circ} \times 0.25^{\circ}$ grid resolution.) features. In this stage, all of the segmented cloud patches are classified into 400 groups based on the similarity of cloud features using the SOFM clustering technique. In the next stage, the rainfall data are coregistered and are assigned into the classified patch groups. The concurrent infrared images and rainfall data in each classified patch group are further sorted, based on a probability matching method. Last, the parameters of the nonlinear fitting function [Eq. (3)] are calculated, based on the sorted pixel infrared and rainfall data. The PERSIANN CCS eventually includes 23 cloud features in the input layer and 400 cloud groups arranged in a $20 \times 20$ matrix in the classification layer, with each group being assigned its unique $T_{b}-R$ function.

\section{b. Insights provided by the calibration results}

The SOFM clustering technique allows exploration of the classified cloud-patch features through its twodimensional feature map. Figure 5a shows the $20 \times 20$ groups arranged in two-dimensional coordinates. Each classified group is described by a set of input patch features. The status of the cloud patch can be explained by listing the input features on each classified group (see Figs. 5b-d). Figure 5b, for instance, shows the contour map of cloud-patch minimum temperatures $\left(T_{\min }\right)$ on $20 \times 20$ groups. Cloud-patch groups in the upperright corner are associated with warmer clouds where cloud-patch $T_{\min }$ are higher than $235 \mathrm{~K}$. Following the arrow, the cloud patch's $T_{\min }$ are gradually reduced, and the patches in the lower-right corner are associated with cold clouds, where their $T_{\min }$ are lower than $205 \mathrm{~K}$.

The contour plot of TOPG on $20 \times 20$ groups in Fig. $5 \mathrm{c}$ shows the cloud patch's temperature gradient near the coldest pixel. This index helps us to locate those cloud-patch groups having strong upwelling movement near the coldest convective core area. Heavy convective clouds usually present tight temperature gradients near the cold core area. They are marked with an " $X$ " in Fig. 5c. An interesting point that should be highlighted is an observation that was made when Figs. $5 \mathrm{c}$ and $5 \mathrm{~b}$ were compared with respect to the location of the $\mathrm{X}$ marks. Note that those high-TOPG patch groups cover a wide range of temperatures, suggesting that convective clouds are not exclusive to the cold cloud regions.

Next we examine the contour plot of the local texture variation [the mean value of the local standard deviation 
TABLE 4. Comparison of daily rainfall estimates from PERSIANN CCS and radar observation on 8-9 Jul 1999.

\begin{tabular}{|c|c|c|c|c|c|c|c|c|}
\hline Spatial scales & No. pixels & Rmse (mm) & Ratio & CORR & SKILL & POD & FAR & CSI \\
\hline \multicolumn{9}{|c|}{8 Jul 1999: $25^{\circ}-45^{\circ} \mathrm{N}, 100^{\circ}-120^{\circ} \mathrm{W}$; mean observed rainfall $3.8 \mathrm{~mm}$ day $^{-1}$} \\
\hline $0.04^{\circ} \times 0.04^{\circ}$ & 166190 & 6.93 & 1.09 & 0.613 & 0.655 & 0.850 & 0.154 & 0.769 \\
\hline $0.12^{\circ} \times 0.12^{\circ}$ & 17921 & 5.32 & 1.07 & 0.676 & 0.690 & 0.865 & 0.109 & 0.821 \\
\hline $0.24^{\circ} \times 0.24^{\circ}$ & 4387 & 4.44 & 1.06 & 0.721 & 0.710 & 0.896 & 0.080 & 0.854 \\
\hline $0.50^{\circ} \times 0.50^{\circ}$ & 1045 & 3.26 & 1.03 & 0.794 & 0.719 & 0.942 & 0.040 & 0.898 \\
\hline $1.00^{\circ} \times 1.00^{\circ}$ & 210 & 2.25 & 1.015 & 0.880 & 0.750 & 1.000 & 0.010 & 0.970 \\
\hline \multicolumn{9}{|c|}{9 Jul 1999: $25^{\circ}-45^{\circ} \mathrm{N}, 100^{\circ}-120^{\circ} \mathrm{W}$; mean observed rainfall $4.7 \mathrm{~mm}$ day $^{-1}$} \\
\hline $0.04^{\circ} \times 0.04^{\circ}$ & 166190 & 8.37 & 1.13 & 0.608 & 0.612 & 0.837 & 0.221 & 0.721 \\
\hline $0.12^{\circ} \times 0.12^{\circ}$ & 17921 & 6.39 & 1.11 & 0.664 & 0.638 & 0.849 & 0.169 & 0.778 \\
\hline $0.24^{\circ} \times 0.24^{\circ}$ & 4387 & 5.14 & 1.078 & 0.719 & 0.675 & 0.885 & 0.122 & 0.831 \\
\hline $0.50^{\circ} \times 0.50^{\circ}$ & 1045 & 3.79 & 1.063 & 0.785 & 0.697 & 0.950 & 0.080 & 0.875 \\
\hline $1.00^{\circ} \times 1.00^{\circ}$ & 210 & 2.63 & 1.035 & 0.855 & 0.736 & 0.990 & 0.030 & 0.950 \\
\hline
\end{tabular}

of the cloud temperature $\left(\mathrm{MSTD}^{5}\right)$ ] in $20 \times 20$ groups, as shown in Fig. 5d. This feature indicates the average of local standard deviation of pixel temperature in a neighborhood of $5 \times 5$ pixels for all pixels under the cloud-patch coverage. Again, the higher MSTD ${ }^{5}$ groups are marked with an X. Note that patches with a higher MSTD $^{5}$ correspond very closely to a higher TOPG in Fig. 5c. The averaged rain rate in the classified $20 \times$ 20 groups shown in Fig. 5e is assigned based on 1month NCEP gauge-corrected radar data. Cloud patches with higher average rain rates are similarly marked with an $\mathrm{X}$. Note that patches with higher rain rates correspond well to the groups with higher TOPG and MSTD ${ }^{5}$ (Figs. $5 \mathrm{c}-\mathrm{e})$.

Last, we examine the two other feature maps. Figure $5 f$ shows the contour map of cloud-patch size (AREA) on $20 \times 20$ groups, and the total patch rainfall amounts are depicted in Fig. 5g. Patch groups with AREA larger than 3000 pixels are circled. When one compares Figs. 5f and 5g, it is evident that those circled large AREA regions correspond to higher total patch rainfall. Interesting to note is that, when we make a comparison with Fig. 5e, the encircled patches with larger AREA groups do not necessarily correspond to a high rainfall rate, which indicates that a large portion of the cloud patch generates little or no rain in those large AREA groups.

What remains to be done is the transformation of the patch information to the pixel rainfall rate for the areas under the patch coverage. Figure $6 \mathrm{a}$ is intended to provide a visualization of the calibrated $T_{b}-R$ curves for each of the $20 \times 20$ patch groups. Each $T_{b}-R$ curve represents the temperature and rainfall-rate relationship at the pixel level for a classified cloud group. For closer examination, seven categories of cloud groups $(\mathrm{C} 0-\mathrm{C} 6)$ are displayed. Note that the $\mathrm{C} 0$ category is associated with warmer and no-rain cloud patches, whereas the other categories (C1-C6) show significant variations of slope and maximum rain rate for temperatures ranging from $T_{\min }>235 \mathrm{~K}$ (in $\mathrm{C} 1$ and $\mathrm{C} 2$ ), $215 \mathrm{~K}>T_{\min }>$ $235 \mathrm{~K}$ (in $\mathrm{C} 3$ and $\mathrm{C} 4$ ), and $T_{\min }<215 \mathrm{~K}$ in (C5 and C6). The $T_{b}-R$ curves of $\mathrm{C} 0-\mathrm{C} 6$ are unique as shown in Fig. 6b. Table 2 summarizes the input features with respect to the seven highlighted categories, and they are explained in appendix $\mathrm{C}$.

\section{Case study and validation}

Several evaluation criteria were selected to validate PERSIANN CCS. The quantitative accuracy of estimates is evaluated using bias: correlation coefficient (CORR), and root-mean-square error (rmse). The performance of rain/no-rain detection is evaluated by the probability of detection (POD), false-alarm ratio (FAR), skill score (SKILL), and critical success index (CSI). Definitions of these criteria are listed in the report of the third Algorithm Intercomparison Project of the Global Precipitation Climatology Project (Ebert 1996). PERSIANN CCS rainfall estimates are validated on an hourly and daily basis for different spatial $\left(0.04^{\circ}, 0.12^{\circ}\right.$, $0.25^{\circ}$, and $0.5^{\circ}$ ) scales. Two rainfall observation datasets were used in this validation: high-temporal-and-spatialresolution NCEP radar data in section 5a and high-quality daily rain gauge data for both the cold and warm seasons in section 5b. PERSIANN CCS was also compared with the PERSIANN product (Sorooshian et al. 2000). The PERSIANN estimates for 1999 were trained using the instantaneous rain rate derived from the Tropical Rainfall Measuring Mission Microwave Imager (TMI). The PERSIANN products for 2002 were calibrated using the rain rates merged from the SSM/I and TMI.

\section{a. Two-day storm event near Las Vegas, Nevada}

\section{1) Hourly rainfall evaluation}

PERSIANN CCS estimates were compared with those of PERSIANN for a flash-flood storm that occurred on 8 July 1999 in Las Vegas, Nevada, and its surrounding area (approximately $300 \mathrm{~km} \times 300 \mathrm{~km}$ ). This intense storm, monitored form 1400 through 1900 UTC (06001100 LST), reportedly caused the worst flooding of the century in the Las Vegas vicinity, resulting in the loss of two human lives and \$20 million in property damage. 

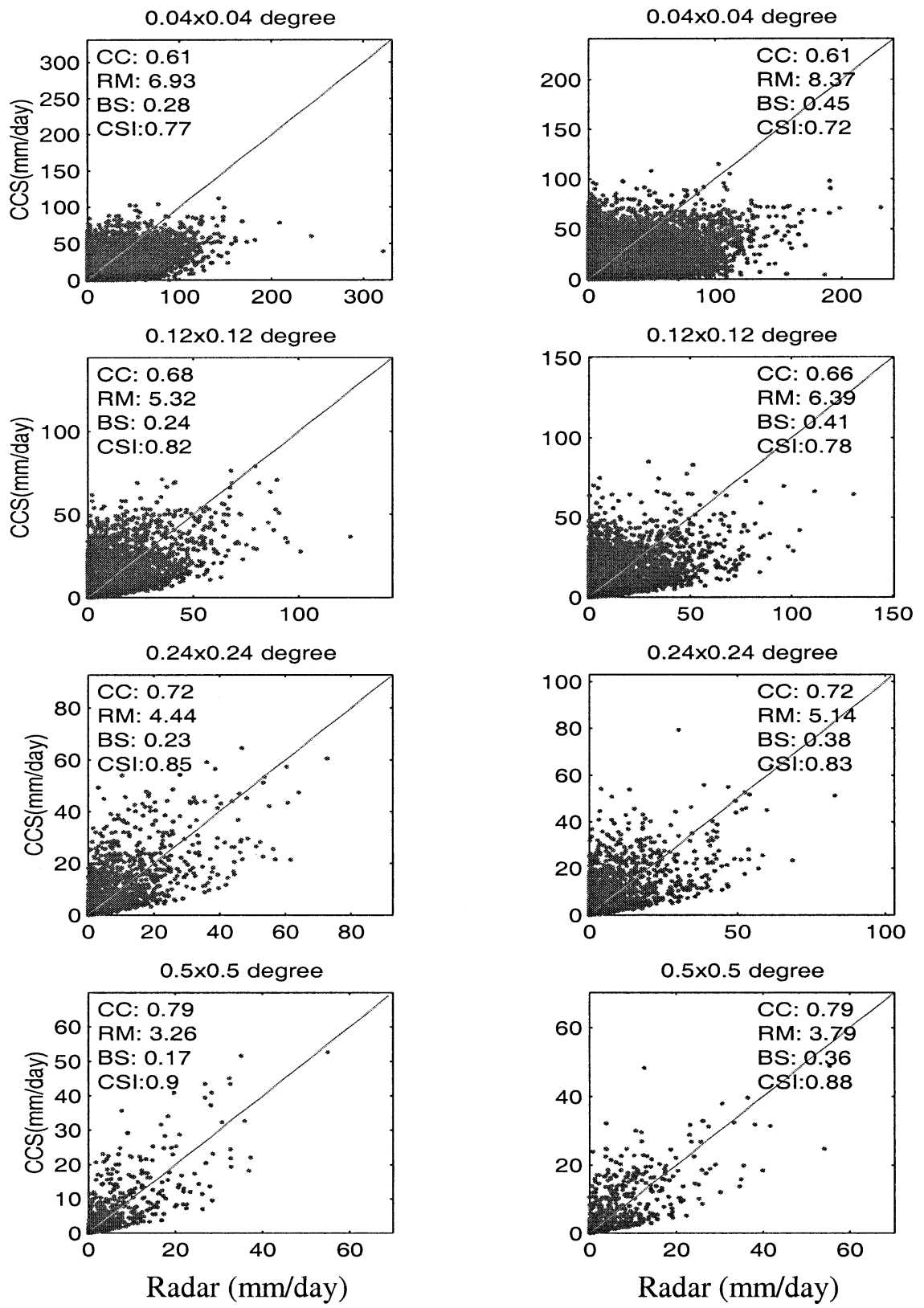

FIG. 8. Scatterplots of radar measurements vs PERSIANN CCS-estimated daily rainfall at four spatial scales for a region located at $25^{\circ}-45^{\circ} \mathrm{N}, 100^{\circ}-120^{\circ} \mathrm{W}$ on (left) $8 \mathrm{Jul}$ and (right) 9 Jul 1999.

TABLE 5. Intercomparison of GPI, PERISANN, and CCS estimation vs gauge-radar data. Note that the sample size for this comparison is 2925 .

\begin{tabular}{|c|c|c|c|c|c|c|}
\hline \multirow[b]{2}{*}{ Daily $0.25^{\circ} \times 0.25^{\circ}$} & \multicolumn{3}{|c|}{8 Jul 1999: $25^{\circ}-45^{\circ} \mathrm{N}, 100^{\circ}-120^{\circ} \mathrm{W}$} & \multicolumn{3}{|c|}{9 Jul 1999: $25^{\circ}-45^{\circ} \mathrm{N}, 100^{\circ}-120^{\circ} \mathrm{W}$} \\
\hline & Rmse (mm) & Ratio & CORR & Rmse (mm) & Ratio & CORR \\
\hline GPI & 14.54 & 0.53 & 0.355 & 15.78 & 0.65 & 0.459 \\
\hline PERSIANN & 6.89 & 1.17 & 0.540 & 7.27 & 1.21 & 0.605 \\
\hline PERSIANN CCS & 4.35 & 1.02 & 0.754 & 5.06 & 1.05 & 0.725 \\
\hline
\end{tabular}



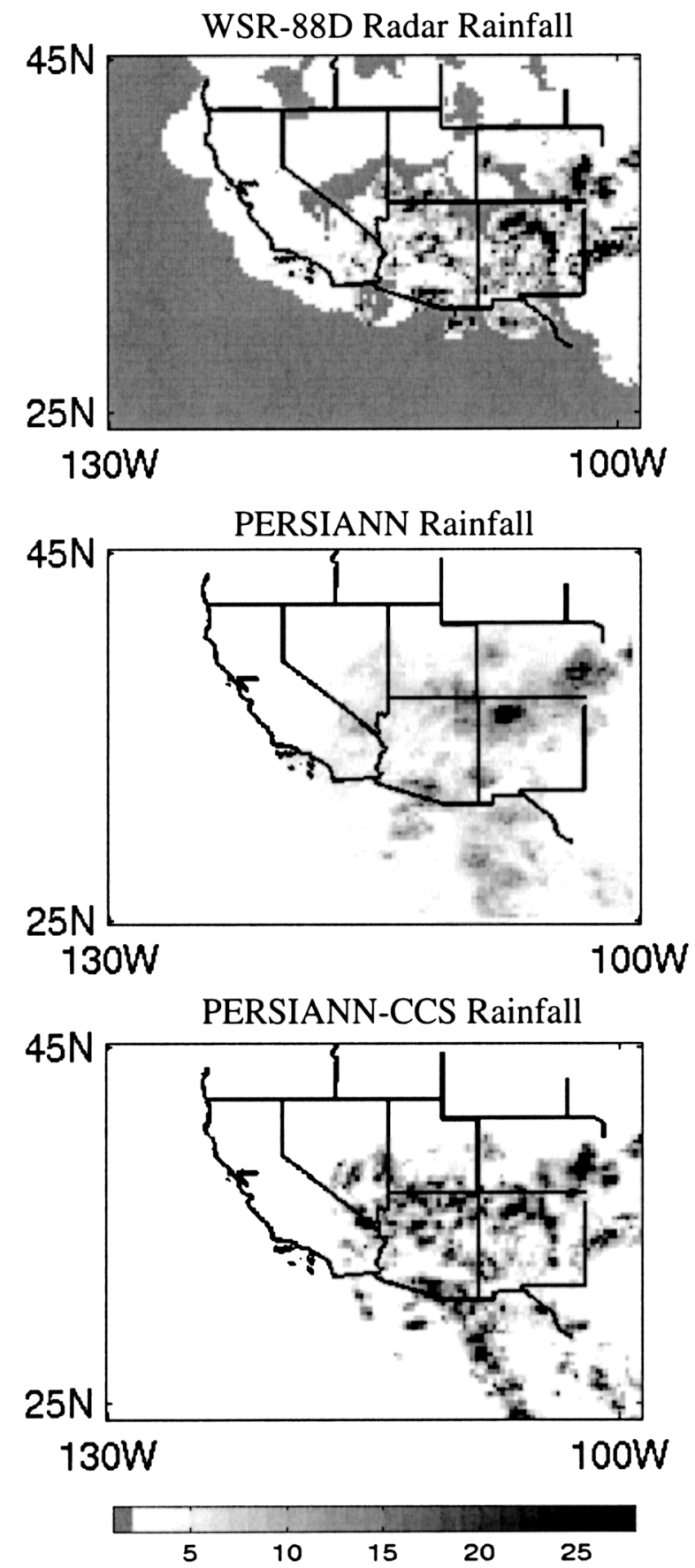

FIG. 9. Comparison of daily rainfall from radar, PERSIANN, and PERSIANN CCS estimates at $0.25^{\circ} \times 0.25^{\circ}$ on $9 \mathrm{Jul} 1999$.

At the instantaneous time scale (at $0.25^{\circ} \times 0.25^{\circ}$ spatial resolution), PERSIANN CCS estimates show a substantial improvement in accuracy and the detection of rain/ no-rain pixels and compared with PERSIANN for this storm (Table 3). A time series of rain-rate and evaluation indices from satellite and radar data is shown in Fig. 7. This result illustrates the advantage of PERSIANN CCS in capturing the high variability in short-term rainfall.

\section{2) DAILy RAINFALl EVALUATION}

(i) Daily comparison of PERSIANN CCS and radar rainfall

Hourly rainfall on 8-9 July 1999 was simulated over a relatively large area $\left(25^{\circ}-45^{\circ} \mathrm{N}, 100^{\circ}-130^{\circ} \mathrm{W}\right)$ and then was accumulated to daily rainfall for model evaluation. PERSIANN CCS was evaluated at $0.04^{\circ}, 0.12^{\circ}$, $0.24^{\circ}, 0.5^{\circ}$, and $1^{\circ}$ resolution. Table 4 shows that the PERSIANN CCS's performance improved as the grid resolution changed from high $\left(0.04^{\circ} \times 0.04^{\circ}\right)$ to low $\left(1^{\circ} \times 1^{\circ}\right)$. The estimates show a good fit at the resolution of $0.12^{\circ}$ and lower, with CORR (including 0.0 rainfall grids) of approximately $0.6-0.8$, rmse of 3-7 mm day ${ }^{-1}$, POD of $0.8-0.9$, and FAR of $0.08-0.17$. The bias ratio was approximately $1.11-1.02$ for the estimates at a resolution of $0.04^{\circ}-1.0^{\circ}$, indicating a minor bias.

Figure 8 shows the scatterplots of PERSIANN CCS estimates against radar observations. The PERSIANN CCS tends to underestimate high rainfall at a small spatial scale $\left(0.04^{\circ}\right)$. When the resolution is reduced to $0.12^{\circ}$ and $0.24^{\circ}$, the underestimation of high rainfall is improved.

\section{(ii) Daily comparison of PERSIANN CCS with PERSIANN}

A comparison of PERSIANN CCS with GPI (the widely used) and PERSIANN results, after remapping at a daily $0.25^{\circ} \times 0.25^{\circ}$ scale, is shown in Table 5 . PERSIANN CCS outperformed PERSIANN on both test days, especially for CORR, which improves from 0.54 to 0.75 on 8 July and from 0.605 to 0.72 on 9 July. Figure 9 shows that PERSIANN estimates were less varied in spatial distribution than those of PERSIANN CCS. Part of the reason for this result is that radar rainfall and PERSIANN CCS are computed at the $0.04^{\circ}$ grid scale but are remapped to a PERSIANN elemental resolution of $0.25^{\circ}$ for comparison. Both PERSIANN CCS and PERSIANN show statistically significant improvement over GPI, particularly in the correlation and bias ratio.

\section{3) PAtch Rainfall VAlidation}

PERSIANN CCS is a patch-based approach, which starts with patch segmentation and classification, followed by the probability matching of the $T_{b}-R$ data distribution and subsequent derivation of an optimized nonlinear $T_{b}-R$ data relationship for each classified cloud patch. During the evolution of a convective cloud, the $T_{b}-R$ distribution varies greatly from the initial stage to the dissipated stage of the cloud patch. Figure 10a illustrates the evolution of a convective cloud patch from 1430 through 2345 UTC 9 July 1999. Figure 10b shows a good fit between the $T_{b}-R$ curves generated from the PERSIANN CCS model and the data dots averaged from infrared images and radar rainfall at each 
time step. Figure 10c shows the time series of the coldest temperature $\left(T_{\min }\right)$ and cloud-area coverage of the cloud patch. Patch-averaged pixel rain rate and patch rainfall derived from PERSIANN CCS are compared with radar (Fig. 10d). As shown in Fig. 10c, this convective cloud has one major growing stage that peaked at the sixth time step, resulting in maximum rain intensity. As shown in Figs. 10c,d, the time step of maximum patch rain intensity matches well with the time step of minimum cloud-patch temperature. Likewise, the time step of maximum patch rainfall volume associates well with the time step of maximum patch area. The case demonstrated in Fig. 10 is unique, with the cloud patches clearly separable. For cases in which multiple convective storms are mixed together (not shown here), the time evolution of the patch-averaged pixel rain intensity and patch rainfall to the selected features may be more complicated.

Figure 10a shows the evolution of the cloud patch from the beginning (small, warm), through the pre-mature (midsized, cold), mature (maximum sized, coldest), dissipating (midsized, cold), and disappearing life stages. Note that the corresponding $T_{b}-R$ functions in Fig. $6 \mathrm{a}$ also change, in sequence, from cluster 2 , through clusters $4,6,3,1$, and 0 for each life stage, respectively. This result clearly shows the temporal correspondence between cloud features (coldness, geometry, and texture) and precipitation characteristics (rainfall volume and intensity) at every life stage of the cloud patch.

As shown in Fig. 10d, the correlation coefficients of the observed and estimated patch intensity and volume time series are 0.96 and 0.93 , respectively. The bias ratios are around 1.0, showing that the biases of the estimates are low. The fitting curves, as shown in Figs. $10 \mathrm{a}, \mathrm{b}$, imply that the $T_{b}-R$ distribution varies with respect to the cloud patch in different stages and cannot fit well if using a single fixed $T_{b}-R$ function.

Figure 11 shows the curve fitting of the $T_{b}-R$ distribution using PERSIANN CCS and several other approaches. The pixel-to-pixel $T_{b}-R$ relationship is plotted in Fig. 11a and shows a large variation between the cloud-top temperature and surface rainfall at the small scale (hourly measurements at $0.04^{\circ}$ grids). Note that only $10 \%$ of the data were randomly selected from the whole training domain samples for this scatterplot, which could represent the distribution of the total dataset. It is evident that the $T_{b}-R$ relationship cannot be fitted well by a single function. Figure $11 \mathrm{~b}$ shows that the testing data are fitted by multiple (400) $T_{b}-R$ curves from the PERSIANN CCS model. Figure 11c illustrates the fitted curves using threshold, linear, or nonlinear regression curves. The PERSIANN CCS model dispatches the $T_{b}-R$ curves based on the specific cloudpatch properties, whereas threshold/linear/nonlinear approaches produce rainfall estimates with only one unique fitting function. For the improvement of the single-function approaches, applications are made to spatial/temporal accumulation of rainfall to lower resolu- tions, such as $1^{\circ} \times 1^{\circ}$ daily/monthly, or are made to rely on temporal adjustment of the $T_{b}-R$ function from other sources of rainfall observations, such as low-orbiting satellite microwave rainfall estimates or surface radar/gauge measurements.

\section{b. Seasonal validation with U.S. daily gauge analyses}

The PERSIANN CCS estimates were validated using gauge data over the southwestern United States for both the cold and warm seasons. The source of the data is the Climate Prediction Center Real-Time Daily Gauge Analysis (Higgins et al. 2000), which is composed of 5000 National Weather Service stations and several hundred stations per day from the Climate Anomaly Data Base. This dataset has undergone quality control (duplicate station check, buddy check, and standard deviation check against climatological mean) and has been objectively analyzed at a $0.25^{\circ}$ grid scale. The validation of PERSIANN CCS was conducted over the continental United States within $25^{\circ}-45^{\circ} \mathrm{N}, 100^{\circ}-125^{\circ} \mathrm{W}$ during a cold month (February 2002) and a warm season (JuneAugust 2002). All datasets were gridded into the same resolution (daily $0.25^{\circ}$ ). To ensure quality control, only days with more than 500 effective grids are considered; otherwise, no statistical comparison is made.

\section{1) TIME SERIES OF STATISTICAL COMPARISONS FOR} BOTH THE COLD AND WARM SEASONS

Time series of statistical comparisons of daily U.S. rain gauge analyses with PERSIANN and PERSIANN CCS estimates are illustrated in Fig. 12. PERSIANN CCS compares more favorably than PERSIANN with the gauge analyses in all statistics during both the cold (Fig. 12a) and the warm (Fig. 12b) seasons. Both algorithms exhibit similar variability in the time series of the statistics in the two seasons; however, the flatter curves of statistics indicate that PERSIANN CCS performs in a more stable manner, particularly for CORR, SKILL, POD, and FAR.

As shown in Table 6, both algorithms perform better in the warm season than in the cold season, possibly because of the absence of snow cover-screening procedures in the two algorithms or the contamination of cold cirrus clouds during the cold season.

\section{2) HiSTOGRAM OF RAIN RATES FOR THE COLD AND WARM SEASONS}

The distributions of rain rates among the rain gauge, PERSIANN, and PERSIANN CCS during the two seasons are depicted in Figs. 13a and 13b, respectively. During the cold season (Fig. 13a), both algorithms overdetect areas at low rainfall intensities. PERSIANN CCS slightly underdetects areas starting at $22 \mathrm{~mm}$ day $^{-1}$ and upward; PERSIANN starts to converge with gauge analyses at $32 \mathrm{~mm} \mathrm{day}^{-1}$ and substantially un- 
(a) Cloud patch evolution

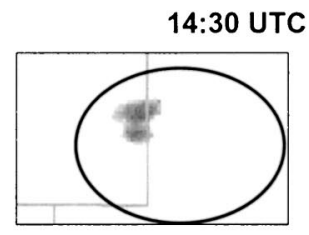

18:30 UTC
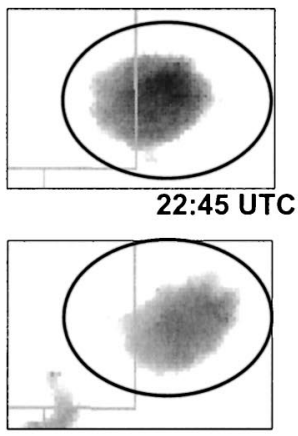

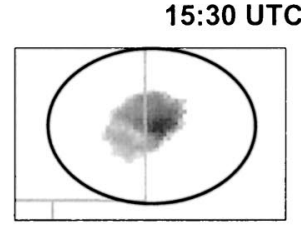

19:00 UTC

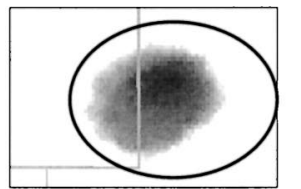

23:15 UTC

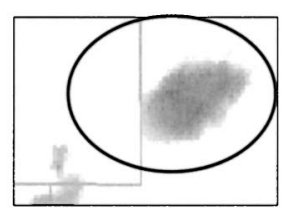

July 91999

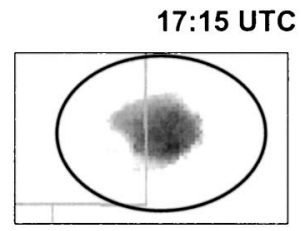

19:30 UTC

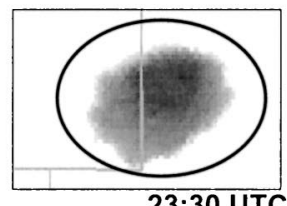

23:30 UTC

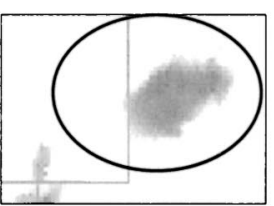

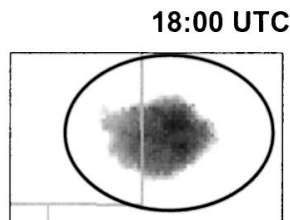

22:00 UTC

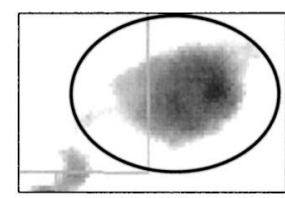

23:45 UTC

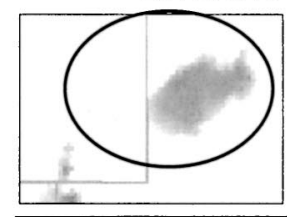

$200 \quad 225 \quad 250 \quad 275 \quad 300$

(b) IR temperature and rainfall $\left(\mathrm{T}_{\mathrm{b}}-\mathrm{R}\right)$ curves changing along with cloud evolution (X axis- $\mathrm{K}$ and $\mathrm{Y}$ axis-mm $\mathrm{hr}^{-1}$ )
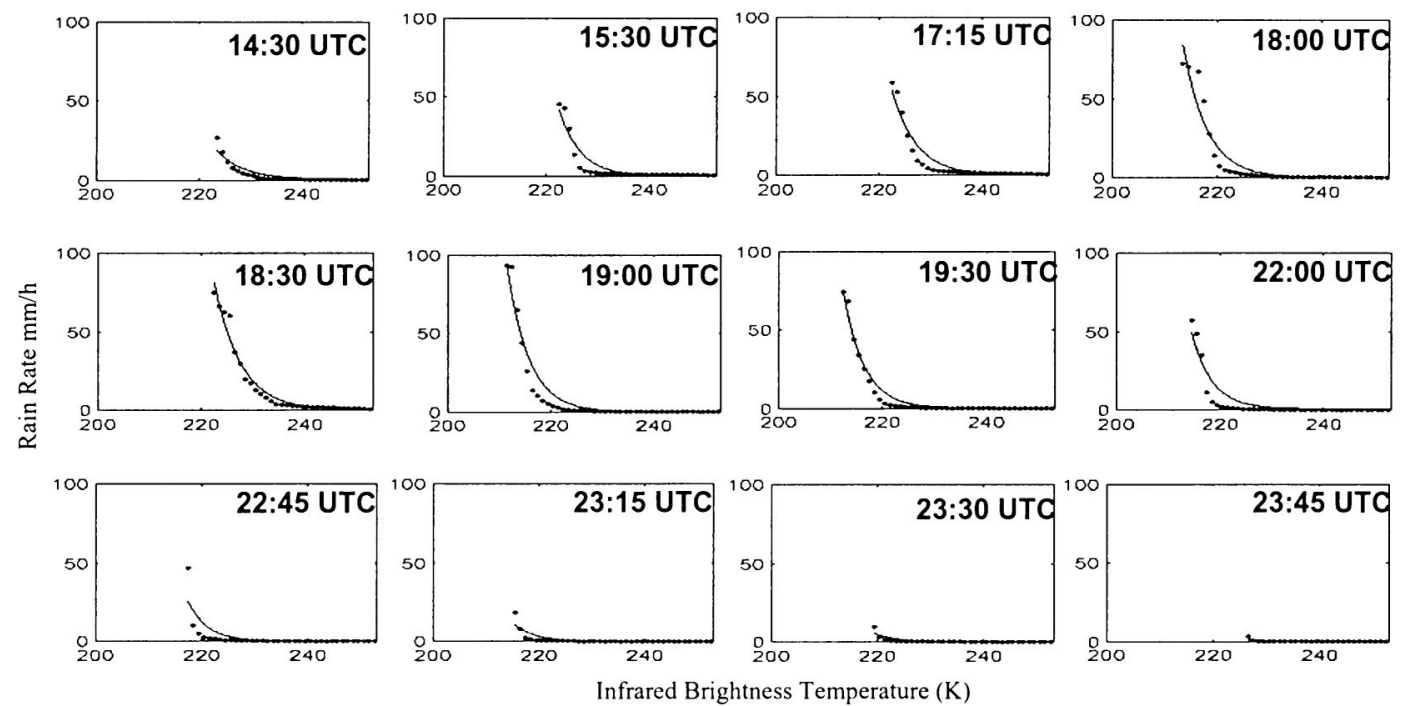

FIG. 10. Cloud-patch rainfall event from 1430 to 2345 UTC 9 Jul 1999 over a region centered at $37^{\circ} \mathrm{N}, 104^{\circ} \mathrm{W}$ : (a) cloud evolution from beginning to end (only partial stages are shown), (b) the corresponding rainfall estimation curves along with the cloud development (dots are the $T_{b}$-rain rate data pairs after being averaged at every $0.5-\mathrm{K}$ interval; curves are the nonlinear functions regressed from the data pairs), (c) time series of cloud-patch minimum pixel temperature and patch size, and (d) time series of patch-averaged pixel rain rate and patch total rainfall estimated from PERSIANN CCS and radar.

derestimates rain rates at $45 \mathrm{~mm}$ day $^{-1}$ and beyond. As shown in Fig. 13b, during the summer season both algorithms detect more areas with rainfall when compared with the gauge analyses. PERSIANN CCS incrementally converges with gauge analyses at high rain rates over $30 \mathrm{~mm} \mathrm{day}^{-1}$; PERSIANN overdetects areas with high rainfall intensity. Note that both algorithms share a very similar distribution in the summer season and exhibit higher variation in February, possibly due to many fewer counting samples in 1-month data samples. The distributions of both satellite rainfall estimates in the summer season more closely correlate with 
(c) Time series of cloud-patch minimum pixel temperature and patch size.
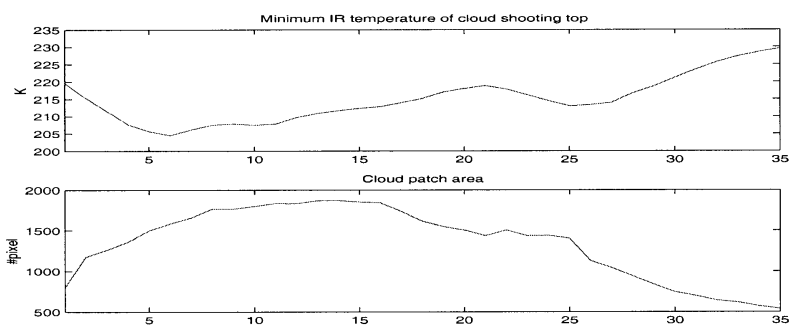

(d) Time series of patch-averaged pixel rain rate and patch total rainfall estimated from PERSIANN-CCS and radar.
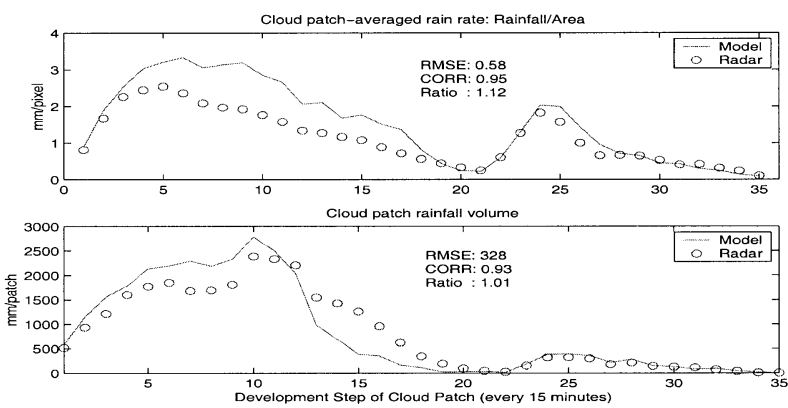

FIG. 10. (Continued)

gauge data than in the cold season. When compared with PERSIANN, PERSIANN CCS demonstrates better agreement with the rain gauge data in the plot of rainfall rates, especially in high-rainfall intensity regions (above $22 \mathrm{~mm} \mathrm{day}^{-1}$ ).

\section{c. The t test for "correlated correlations" between PERSIANN and CCS}

As shown in Tables 3, 5, and 6, PERSIANN CCS has higher correlations than PERSIANN when compared with gauge/radar observations. The question is whether the difference between the correlations is statistically significant or is just sampling variation. A $t$ test for correlated correlations is needed because the two correlations being compared share a variable (rainfall observation, in this case). The long-time standard $t$ test for correlated correlations is Hotelling's $t$ test (Meng et al. 1992).

Given three datasets 1, 2, and 3, R12 indicates the correlation coefficient between data 1 and 2 , and the same for R13 and R23. Hotelling's $t$ test is used to show whether R12 is significantly different from R13. The test statistic is as follows: (a)

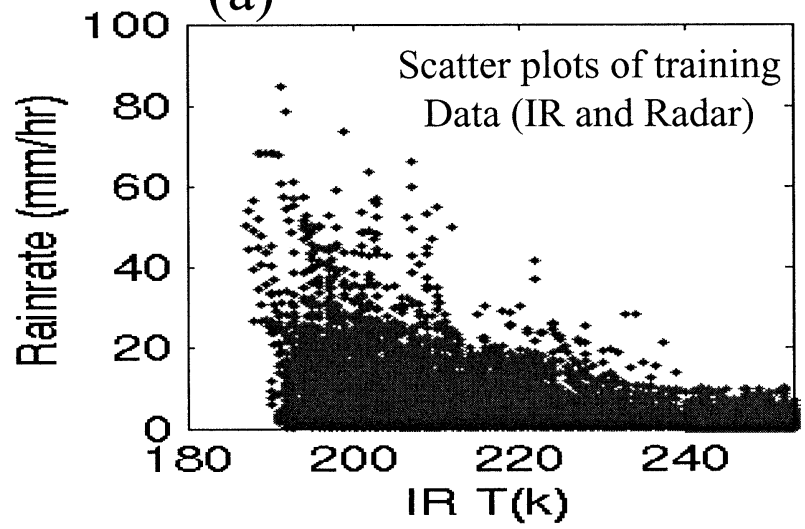

(b)

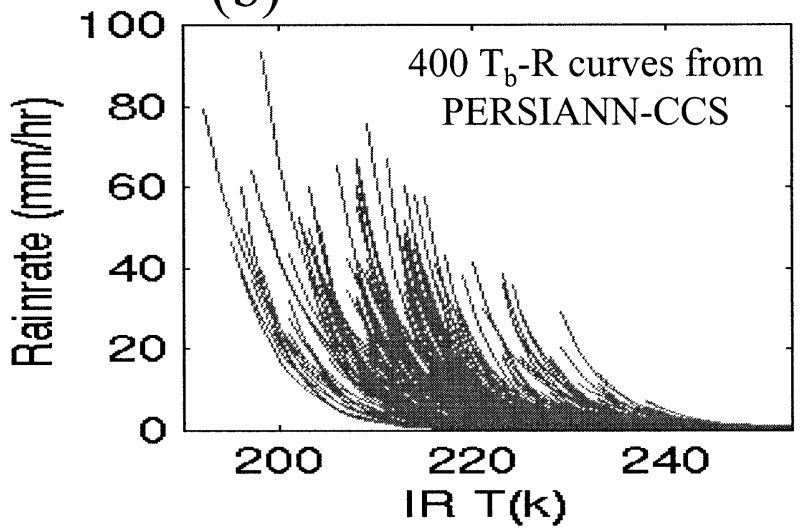

(c)

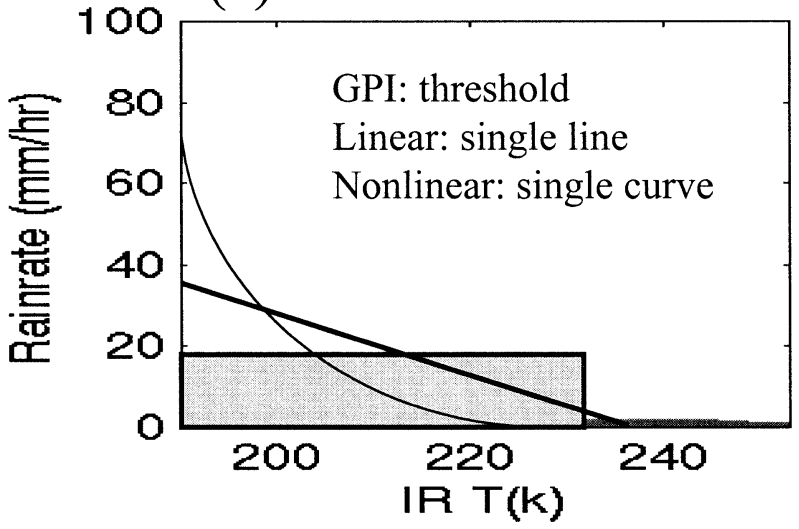

FIG. 11. Multiple-curve-fitting model (PERSIANN CCS) vs singlecurve-fitting models: (a) the scatterplots of cloud-top pixel temperature and radar rain rates from a set of training data, (b) the $T_{b}-R$ curves fitted by the PERSIANN CCS model, and (c) the $T_{b}-R$ curves from several fixed function models (constant threshold, linear, or nonlinear fitting functions).

$$
t=\frac{(\mathrm{R} 12-\mathrm{R} 13) \times \operatorname{sqrt}[(N-3) \times(1+\mathrm{R} 23)]}{\operatorname{sqrt}\left\{2 \times\left[1-(\mathrm{R} 23)^{2}-(\mathrm{R} 12)^{2}-(\mathrm{R} 13)^{2}+(2 \times \mathrm{R} 12 \times \mathrm{R} 13 \times \mathrm{R} 23)\right]\right\}},
$$


(a) February 2002
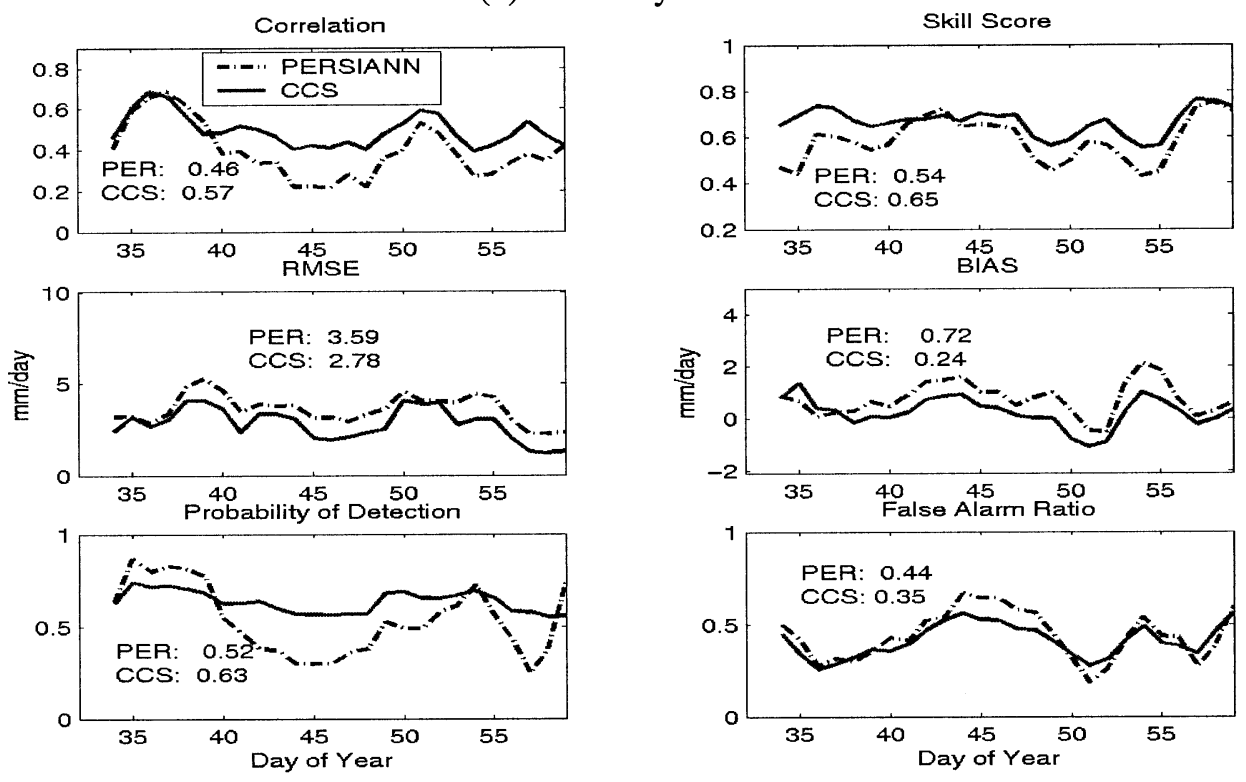

(b) June, July, and August 2002
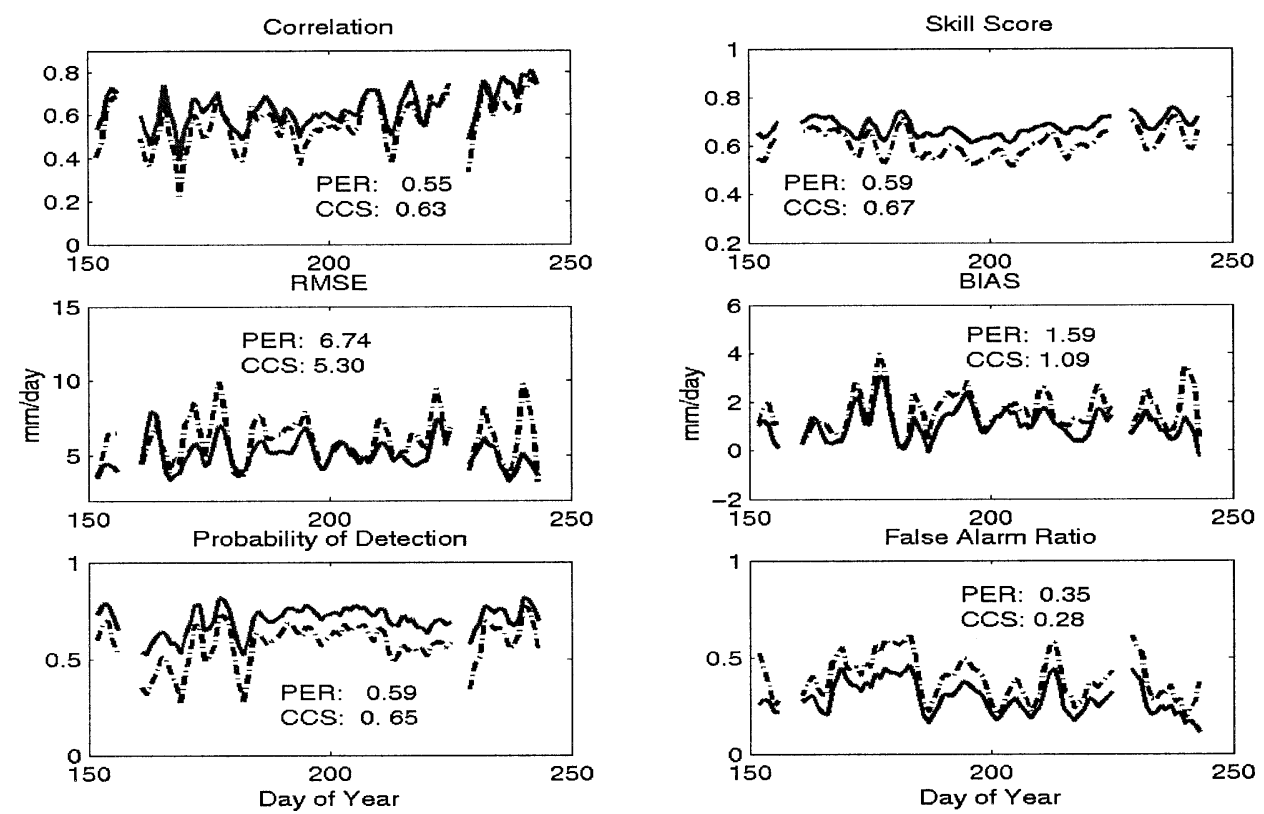

FIG. 12. Time series of selected statistics evaluated at daily $0.25^{\circ}$ scale over $25^{\circ}-45^{\circ} \mathrm{N}, 100^{\circ}-125^{\circ} \mathrm{W}$ during (a) Feb 2002 and (b) Jun-Aug 2002.

TABLE 6. Averaged time series of statistical comparisons of daily rain gauge data with PERSIANN and PERSIANN CCS estimates for both cold and warm seasons.

\begin{tabular}{llcccccc}
\hline \hline & Algorithms & CORR & SKILL & Rmse & Bias & POD & FAR \\
\hline Cold season & PERSIANN & 0.46 & 0.54 & 3.59 & 0.72 & 0.52 & 0.44 \\
& PERSIANN CCS & 0.57 & 0.65 & 2.78 & 0.24 & 0.63 & 0.35 \\
Warm season & PERSIANN & 0.55 & 0.59 & 6.74 & 1.59 & 0.59 & 0.35 \\
& PERSIANN CCS & 0.63 & 0.67 & 5.30 & 1.09 & 0.65 & 0.28 \\
\hline
\end{tabular}


(a) February 2002
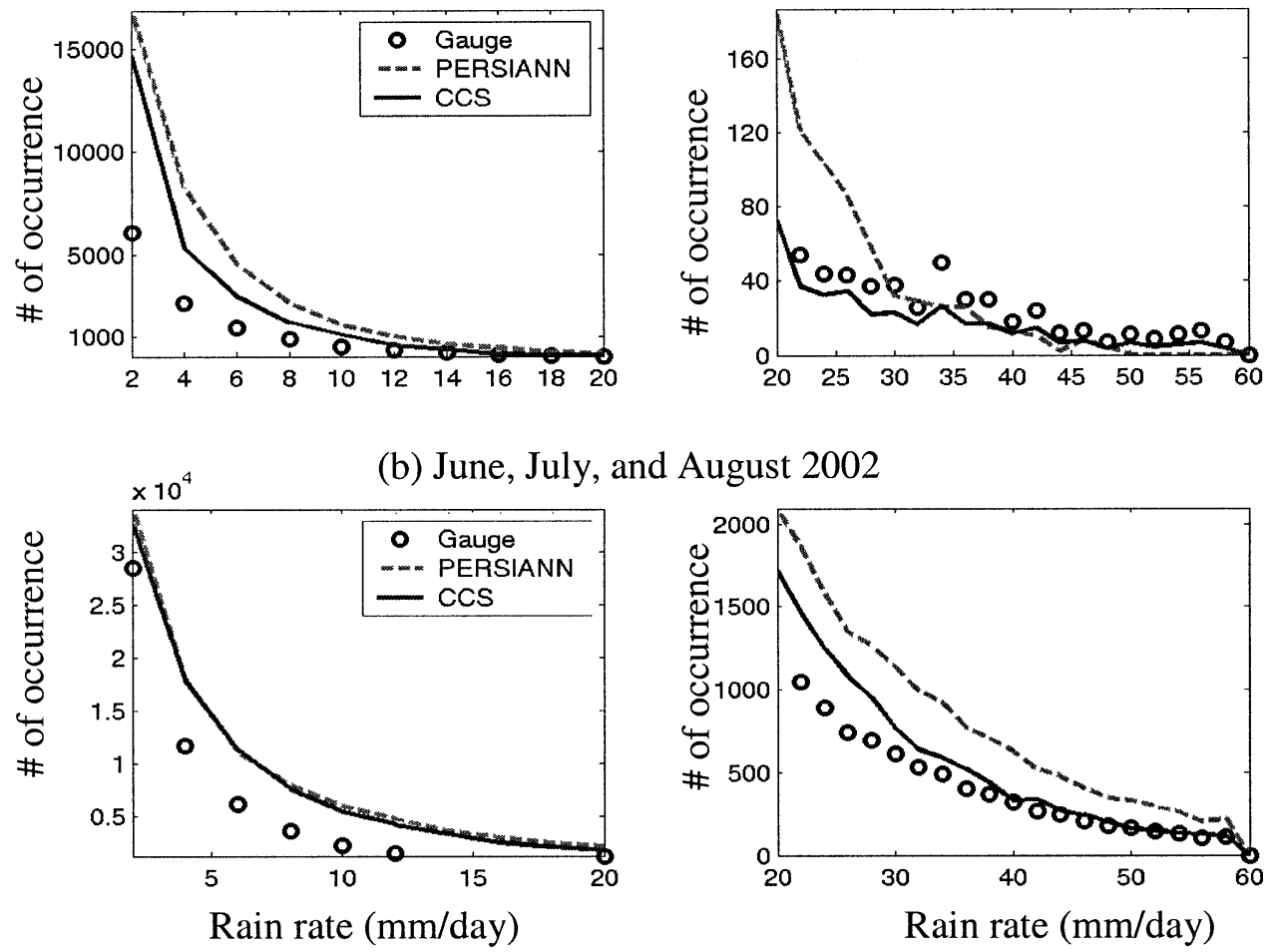

FIG. 13. Plots of rain rates (mm day ${ }^{-1}$ ) during (a) Feb 2002 and (b) Jun-Aug 2002 for (left) rain rates from 0 to $20 \mathrm{~mm} \mathrm{day}^{-1}$ and (right) rain rates from 20 to $60 \mathrm{~mm} \mathrm{day}^{-1}$, both in $2 \mathrm{~mm} \mathrm{day}^{-1}$ increments. Numbers on the $x$ axis represent rain-rate boundaries, and numbers on the $y$ axis are the number of rainrate occurrences. The validation region is the same as in Fig. 12.

where $N$ is the sample size; $t$ has a Student's $t$ distribution with degree of freedom $N-3$.

Given significant level $\alpha=0.05$ and $N \geq 120, \mathrm{R} 12$ is significantly different from R13 if $|t|>t_{\alpha}$, where $t_{\alpha}(=1.65)$ is a critical value from the $t$ table. This test was applied in the comparison cases in Tables 3, 5, and 6 , respectively. The results (Table 7 ) reveal that the correlations of PERSIANN CCS are significantly improved from the correlations of PERSIANN when compared with gauge/radar rainfall.

\section{Summary, conclusions, and future work}

To date, numerous applications of artificial neural networks to solve large-scale problems have been reported in the literature and have provided impressive performances over conventional techniques (Govindaraju et al. 2000). In the case of precipitation, an ANN model, the PERSIANN system, was developed (Hsu et al. 1997, 1999), using the modified counter propagation network, which consists of an SOFM layer and a linear output layer to fit the pixel cloud-top temperature to rain rates. The PERSIANN system estimates surface rainfall at a $0.25^{\circ}$ latitude $\times 0.25^{\circ}$ longitude grid every $30 \mathrm{~min}$ and can accommodate a resolution of $1^{\circ} \times 1^{\circ}$ on a daily scale in various applications (Sorooshian et al. 2000).
One of the limitations of the PERSIANN architecture is that it estimates rainfall based on local pixel information. To overcome this limitation and to extend PERSIANN's ability to utilize additional satellite imagery information from the local pixel scale to cloud-patch scale, PERSIANN CCS is developed. The structural and functional improvements of PERSIANN CCS over PERSIANN are

1) a cloud segmentation procedure that is designed to preprocess IR imagery into a large number of separated cloud patches through an incremental temperature threshold algorithm, as shown in Fig. 1 and appendix A;

2) a feature extraction scheme that retrieves both local pixel temperature textures and regional cloud-patch features instead of only local pixel features as in PERSIANN;

3) a cloud classification algorithm (SOFM) that clusters cloud patches into $400(20 \times 20$ matrix $)$ groups, based on the cloud-patch features extracted from 230-, 235-, and 253-K temperature levels, respectively; and

4) a cloud-rainfall fitting scheme that calibrates different $T_{b}-R$ functions for each classified cloud cluster instead of a single mapping function in PERSIANN. 
TABLE 7. The significance test of correlation between PERSIANN and PERSIANN CCS in comparison with gauge/radar observation. Given significance level $\alpha=0.05, t_{\alpha}(=1.65)<t$.

\begin{tabular}{|c|c|c|c|c|c|}
\hline \multirow[b]{3}{*}{ Case } & \multirow{3}{*}{$\begin{array}{l}\text { Table } 3 \\
\text { Storm }\end{array}$} & \multirow{2}{*}{\multicolumn{2}{|c|}{ Table 5 (1999) }} & \multicolumn{2}{|c|}{ Table 6 (2002) } \\
\hline & & & & \multirow[b]{2}{*}{ Cold season (Feb) } & \multirow{2}{*}{$\begin{array}{c}\text { Warm season } \\
\text { (Jun-Aug) }\end{array}$} \\
\hline & & $8 \mathrm{Jul}$ & 9 Jul & & \\
\hline$N$ & 1890 & 2925 & 2925 & 15724 & 45943 \\
\hline$t$ & 7.46 & 23.15 & 14.67 & 18.05 & 27.73 \\
\hline
\end{tabular}

Another significant feature of the new version is its improved spatial resolution, in which the PERSIANN CCS computation pixel is at $0.04^{\circ} \times 0.04^{\circ}$ as compared with that of PERSIANN at $0.25^{\circ} \times 0.25^{\circ}$.

The performance of the algorithm was evaluated using daily gauge and hourly radar rainfall at various spatial scales $\left(0.04^{\circ}, 0.12^{\circ}, 0.24^{\circ}, 0.25^{\circ}\right.$, and $\left.0.5^{\circ}\right)$. The results show that PERSIANN CCS, because of its comprehensive cloud-patch features and its ability to address the variability of rainfall distributions in different cloud clusters, outperforms PERSIANN. In addition, PERSIANN CCS offers insights in explaining the classified patch features with respect to their pixel rainfall distributions. PERSIANN CCS can be used not only as a finescale rainfall estimation scheme, but also as an explanatory tool to analyze the cloud-rainfall system.

Our evaluation of PERSIANN CCS so far has focused on rainfall estimation over land, where ground-based radar/gauge measurements are available to quantify the rainfall distribution. We are currently extending this work to evaluate PERSIANN CCS's performance over the ocean.

Acknowledgments. Partial support for this research is from NSF STC for "Sustainability of Semi-Arid Hydrology and Riparian Areas" (SAHRA) (Grant EAR9876800), NASA-EOS (Grant NA56GPO185), and TRMM (Grant NAG5-7716). We are grateful to Dr. R. Adler and others of NASA GSFC for their valuable suggestions. The authors also thank the anonymous reviewers and editor for their constructive comments.

\section{APPENDIX A}

\section{The Processing Steps of the ITT Algorithm}

The incremental temperature threshold algorithm segments cloud IR images starting from the coldest pixels to warmer thresholds, controlled by the following steps.

Step 1: Locate pixels with the coldest temperature $T_{\min }$ and local minimum temperatures (initializing seeds). Compute the incremental thresholds using the following:

$$
\mathrm{THT}_{m}=T_{\min }+\Delta T i, i=1,2, \ldots, M,
$$

where

$$
M=\left\{\left(T_{u}-T_{\min }\right) / \Delta T\right\}_{\text {int }} .
$$

Here $T_{\min }$ is the pixel temperature minimum, $T_{u}$ is the temperature that discriminates between a cloudy and clear sky, $\Delta T$ is temperature interval equal to $3 \mathrm{~K}$, and \{\}$_{\text {int }}$ takes the integer values.

Step 2: Grow seeded regions from $\mathrm{THT}_{m}$ to $\mathrm{THT}_{m+1}$. Assuming that at step $m$ there are $n_{1}$ seeded regions covered by temperatures lower than $\mathrm{THT}_{m}$ (the seeded region $A_{i}$ is numerically labeled as $i=1$, $\left.2, \ldots, n_{1}\right)$ :

$$
A^{m}=\left\{A_{1}^{m}, A_{2}^{m}, \ldots, A_{i}^{m}, \ldots, A_{n 1}^{m}\right\} .
$$

The coldest top temperature for each cloud region is

$$
\mathrm{CT}_{i}=\min _{(x, y) \in A_{i}^{m}}[g(x, y)],
$$

where $g(x, y)$ is the temperature of the pixel $(x, y)$. At each step, the algorithm grows first by labeling new seed(s), then by expanding the existing regions $A^{m}$ until reaching the threshold $\mathrm{THT}_{m+1}$. The external boundary EB of the existing regions is

$$
\begin{aligned}
& \mathrm{EB}= \\
& \left\{(x, y) \notin \bigcup_{i=1}^{n} \mathrm{~A}_{i}^{m} \text { and } \mathrm{NB}(x, y) \cap \bigcup_{i=1}^{n} \mathrm{~A}_{i}^{m} \neq \phi\right\},
\end{aligned}
$$

where $\operatorname{NB}(x, y)$ is the set of eight immediate neighbors of the pixel $(x, y)$. If $\operatorname{NB}(x, y)$ connects only to one existing region $\left(A_{i}^{m}\right)$, the pixel $(x, y)$ is merged to region $A_{i}^{m}$; if $\operatorname{NB}(x, y)$ connects to more than one existing region, then this pixel $(x, y)$ is merged to the region $\left(A_{j}^{m}\right)$ that has a minimum temperature difference to the coldest pixel in the region:

$$
\begin{aligned}
j & =\arg _{i} \min \delta_{i}(x, y) \\
& =\arg _{i} \min \left|g(x, y)-\mathrm{CT}_{i}\right| .
\end{aligned}
$$

Repeat the process until the current THT $_{m+1}$ is reached.

Step 3: Find new seeds. A new seed is assigned if a pixel is colder than THT $_{m+1}$ but isolated from any existing region $A^{m}$ :

$$
\begin{aligned}
& \operatorname{Seed}_{\text {new }}^{m}= \\
& \left\{g(x, y)<\operatorname{THT}_{m+1} \text { and } \operatorname{NB}(x, y) \cap \bigcup_{i=1}^{n} \mathrm{~A}_{i}^{m}=\phi\right\} .
\end{aligned}
$$


New seeds are numerically labeled as $n+1, n+$ $2, \ldots, n_{2}$. After completing the $m+1$ iterations, the cloud segments are

$$
\begin{aligned}
& A^{m+1}=\left\{A_{1}^{m+1}, A_{2}^{m+1}, \ldots, A_{i}^{m+1},\right. \\
&\left.\ldots, A_{n 1}^{m+1}, \ldots, A_{n 2}^{m+1}\right\} .
\end{aligned}
$$

Step 4: Repeat steps 2 and 3 until THT $_{m} \geq T_{u}$.

\section{APPENDIX B}

\section{Cloud-Patch Feature Description}

Given a cloud patch with a size of $A_{k}$, total pixel count $N$, and pixel brightness temperature of $g(x, y)$, those patch features listed in Table 1 are described as follows.

1) Minimum temperature of a cloud patch $\left(T_{\min }\right)$ is

$$
T_{\min }=\min _{(x, y) \in A_{k}}[g(x, y)]
$$

2) Mean temperature of a cloud patch $\left(T_{\text {mean }}\right)$ is

$$
T_{\text {mean }}=\sum_{(x, y) \in A_{k}}[g(x, y)] / N \text {. }
$$

3) Cloud-patch area $\left(A_{k}\right)$ is

$$
A_{k}=N \times \text { pixel resolution. }
$$

4) Shape index (SI) is defined as the ratio of the geometric eccentricity of a cloud patch $(I)$ to that of a round patch with the same size $\left(I_{0}\right)$ :

$$
\mathrm{SI}=I / I_{0},
$$

where

$$
I=\sum_{i=1}^{N}\left[\left(x_{i}-G_{x}\right)^{2}+\left(y_{i}-G_{y}\right)^{2}\right],
$$

$\left(x_{i}, y_{i}\right)$ is the coordinate of pixel $i,\left(G_{x}, G_{y}\right)$ is the geometric center of the cloud patch, with

$$
G_{x}=\frac{1}{N} \sum_{i=1}^{N} x_{i} \text { and } G_{y}=\frac{1}{N} \sum_{i=1}^{N} y_{i},
$$

and $I_{0}$ is the geometric inertia of a round patch with the same area. Given a certain size of cloud patch, the rounder-shaped patches give a smaller shape index. A perfectly circular cloud patch has the smallest SI value of 1 .

5) Standard deviation of cloud-patch top temperature (STD) is

$$
\mathrm{STD}=\left\{\sum_{(x, y) \in A_{k}}\left[g(x, y)-T_{\text {mean }}\right]^{2} /(N-1)\right\}^{1 / 2} .
$$

6) Mean value of the local standard deviation of cloudpatch top temperature $\left(\mathrm{MSTD}^{5}\right)$ is

$$
\operatorname{MSTD}^{5}=\sum_{i=1}^{N} \frac{\mathrm{STD}_{i}^{5}}{N},
$$

where $\mathrm{STD}_{i}^{5}$ is the standard deviation of cloud-top temperature with a window size of $5 \times 5$ centered on pixel $i$.

7) Standard deviation of local standard deviation of cloud-top temperature $\left(\mathrm{STD}_{\text {std }}^{5}\right)$ is

$$
\mathrm{STD}_{\mathrm{std}}^{5}=\left[\sum_{i} \frac{\left(\mathrm{STD}_{i}^{5}-\mathrm{MSTD}^{5}\right)}{(N-1)}\right]^{1 / 2} .
$$

8) Temperature gradient of cloud cold core (TOPG) measures the cloud-patch average pixel temperature gradient around the cold core pixel $\left(T_{\min }\right)$ and serves a similar purpose as a temperature slope parameter addressed in Negri et al. (2002) to determine convective cores in order to eliminate nonprecipitating cirrus. The threshold of $15 \mathrm{~K}$ above the coldest pixel $T_{\min }$ is empirically chosen to calculate TOPG from the $T_{\min }$ to the surrounding pixels having a temperature of $T_{\min }+15 \mathrm{~K}$ :

$$
\text { TOPG }=\sum_{i=1}^{N} \frac{15}{\mu(i)} / N,
$$

where $N$ is the number of pixels along the border of $T_{\min }+15 \mathrm{~K}$ and $\mu(i)$ is the distance from the border pixel $i$ to the coldest pixel.

9) Gray-image texture [maximum angular second moment (MASM)] information is characterized by a set of gray-level co-occurrence matrices $H_{d, \phi}$, with the element $h(i, j)_{d, \phi}$ as the estimate of the probability that gray level $i$ and gray level $j$ are located at a distance $d$ and angle $\phi$, with a fixed direction (Haralick et al. 1973). The co-occurrence matrices $H_{d, \phi}$ delineate the texture information of an image on various scales and directions. The angular second moment (ASM) of the co-occurence matrix located at various distances and directions is

$$
\operatorname{ASM}_{d, \phi}=\sum_{i} \sum_{j}\left[h(i, j)_{d, \phi}\right]^{2} .
$$

The ASM features computed at various directions are usually redundant. In our case, only the maximum ASM was selected:

$$
\operatorname{MASM}=\frac{\max }{\phi}\left\{\mathrm{ASM}_{\phi} \mid \phi=0^{\circ}, 45^{\circ}, 90^{\circ}, 135^{\circ}\right\} .
$$

\section{APPENDIX C}

\section{Explanation of the Seven Highlighted Cloud Categories}

The calibrated $20 \times 20 T_{b}-R$ curves (Fig. 6a) form seven highlighted categories (Fig. 6b) noted as C0-C6, and they are explained as follows.

C0: Cloud minimum temperature $\left(T_{\min }\right)$ is higher 
than $245 \mathrm{~K}$, and there is a small cloud-patch size $(<100$ pixels), a low temperature gradient near $T_{\min }$, and mostly no rain. The slope of $T_{b}-R$ is approximately 0 . This category is related to warm, no-rain clouds.

$\mathrm{C} 1$ : The range of the coldest patch pixel temperatures is $235-245 \mathrm{~K}$, and the texture indices are low (TOPG 1; MSTD $^{5} \sim 1$; STD < 4). Cloud-patch size is relatively small, and the slope of the $T_{b}-R$ curve is flat. The cloud-patch average pixel rain rate is less than $0.5 \mathrm{~mm} \mathrm{~h}^{-1}$, and the cloud-patch rainfall is less than $100 \mathrm{~mm} \mathrm{~h}^{-1}$.

C2: The coldest pixel temperature of a cloud patch is similar to that of the $\mathrm{C} 1$ category, but the texture indices are much higher $\left(\mathrm{TOPG} \sim 3\right.$ MSTD $^{5} \sim$ 3.5; STD $\sim 4-8$ ). The slope of the $T_{b}-R$ curve is high, with an average pixel rain rate of around $0.5-$ $1.0 \mathrm{~mm} \mathrm{~h}^{-1}$ and a total rainfall per patch of about $1000 \mathrm{~mm} \mathrm{~h}^{-1}$, which is about 10 times that of the C1 category.

C3: The coldest patch pixel temperature is around 215-235 K, and the texture indices are low (TOPG $\sim 1$; $^{2}$ MSD $^{5} \sim 2.5$; STD $\sim 6-8$ ). The average pixel rain rate is around $0.1-0.5 \mathrm{~mm} \mathrm{~h}^{-1}$, and the accumulated rainfall per patch is around 500-2000 $\mathrm{mm} \mathrm{h}^{-1}$. As shown in Fig. 6b, the slope of the $T_{b}-$ $R$ curve is flat.

C4: The coldest patch pixel temperature is around 215-235 K, and the texture indices are relatively high (TOPG 3.5; MSTD $^{5} \sim 4$; STD 8-10). The average pixel rain rate is around $0.5-1.0 \mathrm{~mm}$ $\mathrm{h}^{-1}$, and the accumulated rainfall per patch is around 1000-2500 $\mathrm{mm} \mathrm{h}^{-1}$. The maximum pixel rain rate can be as high as $60 \mathrm{~mm} \mathrm{~h}^{-1}$.

C5: This category is associated with cold cloud patches with $T_{\min }<215 \mathrm{~K}$ and low texture indices (TOPG $\sim 1.5$; MSTD $^{5} \sim 3$ ). The average pixel rain rate is around $0.5-1.5 \mathrm{~mm} \mathrm{~h}^{-1}$, and the accumulated patch rainfall is around $3000-6000 \mathrm{~mm}$ $\mathrm{h}^{-1}$. The $T_{b}-R$ curve is long and mildly steep.

C6: The coldest patch pixel temperature is lower than $215 \mathrm{~K}$, and the texture indices are relatively high (TOPG $\sim 4.5 ;$ MSTD $^{5} \sim 5$ ). The average pixel rain rate is around $1.5-4.5 \mathrm{~mm} \mathrm{~h}^{-1}$, and the accumulated patch rainfall is around $2000-3500 \mathrm{~mm}$ $\mathrm{h}^{-1}$. This type of cloud patch has a $T_{b}-R$ curve with a steep slope. Maximum pixel rainfall could reach $100 \mathrm{~mm} \mathrm{~h}^{-1}$.

\section{REFERENCES}

Adler, R. F, and A. J. Negri, 1988: A satellite infrared technique to estimate tropical convective and stratiform rainfall. J. Appl. Meteor., 27, 30-51.

—_, and ——, 1993: Estimation of monthly rainfall over Japan and surrounding waters from a combination of low-orbit mi- crowave and geosynchronous IR data. J. Appl. Meteor., 32, 335-356.

Arkin, P. A., and B. N. Meisner, 1987: The relationship between largescale convective rainfall and cold cloud over the Western Hemisphere during 1982-84. Mon. Wea. Rev., 115, 51-74.

Atlas, D., D. Rosenfeld, and D. B. Wolff, 1990: Climatologically tuned reflectivity-rain rate relations and links to area-time integrals. J. Appl. Meteor., 29, 1120-1135.

Ebert, E. E., 1996: Results of the 3rd Algorithm Intercomparison Project (AIP-3) of the Global Precipitation Climatology Project (GPCP). Bureau of Meteorology Centre Res. Rep. 55, 204 pp.

__ and M. J. Manton, 1998: Performance of satellite rainfall estimation algorithms during TOGA COARE. J. Atmos. Sci., 55, 1537-1557.

Govindaraju, R. S., and A. R. Rao, 2000: Artificial Neural Networks in Hydrology. Kluwer Academic, 329 pp.

Griffith, C. G., W. L. Woodley, P. G. Grube, D. W. Martin, J. Stout, and D. N. Sikdar, 1978: Rain estimation from geosynchronous satellite imagery-Visible and infrared studies. Mon. Wea. Rev., 106, 1153-1171

Haralick, R. M., K. Shanmuban, and I. Dinstein, 1973: Texture features for image classification. IEEE Trans. Syst. Man Cybernetics, 6, 610-621.

Higgins, R. W., W. Shi, E. Yarosh, and R. Joyce, cited 2000: Improved United States Precipitation Quality Control System and Analysis. NCEP/CPC Atlas No. 7 [Available online at http://www.cpc. ncep.noaa.gov/research_papers/ncep_cpc_atlas/7/index.html.]

Hsu, K., X. Gao, S. Sorooshian, and H. V. Gupta, 1997: Precipitation estimation from remotely sensed information using artificial neural networks. J. Appl. Meteor., 36, 1176-1190.

- H. V. Gupta, X. Gao, and S. Sorooshian, 1999: Estimation of physical variables from multichannel remotely sensed imagery using a neural network: Application to rainfall estimation. Water Resour. Res., 35, 1605-1618.

$-\_,-,-—,-$ -, and B. Iman, 2002: Self-organizing linear output (SONO): An artificial neural network suitable for hydrologic modeling and analysis. Water Resour. Res., 38, 1302-1318.

Kohonen, T., 1982: Self-organized formation of topologically correct feature maps. Biol. Cybernetics, 43, 59-69.

Meng, X. L., R. Rosenthal, and D. B. Rubin, 1992: Comparing correlated correction coefficients. Psychol. Bull., 111, 172-175.

NCEP, cited 2003: National stage IV QPE product. [Available online at http://wwwt.emc.ncep.noaa.gov/mmb/ylin/pcpanl/stage4/.]

Negri, A. J., T. L. Bell, and L. Xu, 2002: Sampling of the diurnal cycle of precipitation using TRMM. J. Atmos. Oceanic Technol., 19, 1333-1344.

Sorooshian, S., K. Hsu, X. Gao, H. V. Gupta, B. Imam, and D. Braithwaite, 2000: Evolution of PERSIANN system satellite-based estimates of tropical rainfall. Bull. Amer. Meteor. Soc., 81, 20352046.

Vicente, G., R. A. Scofield, and W. P. Mensel, 1998: The operational GOES infrared rainfall estimation technique. Bull. Amer. Meteor. Soc., 79, 1881-1898.

Woodley, W. L., C. G. Griffith, J. S. Griffin, and S. C. Stroomatt, 1980: The inference of GATE convective rainfall from SMS-1 imagery. J. Appl. Meteor., 19, 338-408.

Wu, R., J. A. Weinman, and R. T. Chin, 1985: Determination of rainfall rates from GOES satellite images by a pattern recognition technique. J. Atmos. Oceanic Technol., 2, 314-330.

Xu, L., X. Gao, S. Sorooshian, P. A. Arkin, and B. Imam, 1999a: A microwave infrared threshold technique to improve the GOES precipitation index. J. Appl. Meteor., 38, 569-579.

-, S. Sorooshian, X. Gao, and H. Gupta, 1999b: A cloud-patch technique for identification and removal of no-rain clouds from satellite infrared imagery. J. Appl. Meteor., 38, 11701181 . 